\title{
$\gamma$-Secretase: Successive Tripeptide and Tetrapeptide Release from the Transmembrane Domain of $\beta$-Carboxyl Terminal Fragment
}

\author{
Mako Takami, ${ }^{1}$ Yu Nagashima, ${ }^{2}$ Yoshihisa Sano, ${ }^{3}$ Seiko Ishihara, ${ }^{1}$ Maho Morishima-Kawashima, ${ }^{4}$ Satoru Funamoto, ${ }^{1}$ \\ and Yasuo Ihara ${ }^{1}$ \\ ${ }^{1}$ Department of Neuropathology, Faculty of Life and Medical Sciences, Doshisha University, Kizugawa 619-0225, Japan, ${ }^{2}$ Department of Neurology, Faculty of \\ Medicine, University of Tokyo, Tokyo 113-0033, Japan, ${ }^{3}$ Drug Metabolism and Pharmacokinetics Research Section, Eisai Company Ltd., Tsukuba 300-2635, Japan, \\ and ${ }^{4}$ Department of Molecular Neuropathology, Graduate School of Pharmaceutical Sciences, Hokkaido University, Sapporo 060-0812, Japan
}

Amyloid $\beta$ protein $(\mathrm{A} \beta)$, a pathogenic molecule associated with Alzheimer's disease, is produced by $\gamma$-secretase, which cleaves the $\beta$-carboxyl terminal fragment ( $\beta$ CTF) of $\beta$-amyloid precursor protein in the middle of its transmembrane domain. How the cleavage proceeds within the membrane has long been enigmatic. We hypothesized previously that $\beta$ CTF is cleaved first at the membranecytoplasm boundary, producing two long $\mathrm{A} \beta \mathrm{s}, \mathrm{A} \beta_{48}$ and $\mathrm{A} \beta_{49}$, which are processed further by releasing three residues at each step to produce $\mathrm{A} \beta_{42}$ and $\mathrm{A} \beta_{40}$, respectively. To test this hypothesis, we used liquid chromatography tandem mass spectrometry (LC-MS/MS) to quantify the specific tripeptides that are postulated to be released. Using CHAPSO (3-[(3-cholamidopropyl)dimethylammonio]-2hydroxyl-1-propanesulfonate)-reconstituted $\gamma$-secretase system, we confirmed that $\mathrm{A} \beta_{49}$ is converted to $\mathrm{A} \beta_{43 / 40}$ by successively releasing two or three tripeptides and that $\mathrm{A} \beta_{48}$ is converted to $\mathrm{A} \beta_{42 / 38}$ by successively releasing two tripeptides or these plus an additional tetrapeptide. Most unexpectedly, LC-MS/MS quantification revealed an induction period, 3-4 min, in the generation of peptides. When extrapolated, each time line for each tripeptide appears to intercept the same point on the $x$-axis. According to numerical simulation based on the successive reaction kinetics, the induction period exists. These results strongly suggest that $\mathrm{A} \beta$ is generated through the stepwise processing of $\beta$ CTF by $\gamma$-secretase.

\section{Introduction}

There are three possible explanations of the intramembrane cleavage of $\beta$-carboxyl terminal fragment ( $\beta$ CTF) of amyloid precursor protein (APP) leading to the generation of $\mathrm{A} \beta_{40}$ and $\mathrm{A} \beta_{42}$ : (1) $\varepsilon$-cleavage occurs first and is followed by $\gamma$-cleavage; (2) both cleavages occur at the same time; and (3) $\gamma$-cleavage occurs first and is followed by $\varepsilon$-cleavage. In the first case, we should find longer amyloid $\beta$ protein $(A \beta)$ species (longer than $A \beta_{42}$ ) within the cell, whereas in the third case we should detect longer APP intracellular domain (AICD) (for example, AICD41-99 and AICD43-99, counterparts of $A \beta_{40}$ and $A \beta_{42}$, respectively). Despite extensive searching, we failed to find longer AICDs. In contrast, using a newly developed urea/SDS-PAGE method, we identified distinct spe-

Received May 19, 2009; revised Aug. 12, 2009; accepted Sept. 1, 2009.

This work was supported in part by a Grant-in-Aid for Scientific Research on Priority Areas, Research on Pathomechanisms of Brain Disorders (Y.I.), by a Grant-in-Aid for Scientific Research for the Encouragement of Young Scientists (A) (S.F.) from the Ministry of Education, Culture, Sports, Science, and Technology, and by the grant program "Collaborative Development of Innovative Seeds" (S.F.) from the Japan Science and Technology Agency. We also thank Information-Technology Promotion Agency, Japan and Exploratory IT Human Resources Project (MITOH Program) for kind support (Y.N.). We thank Takeda Chemical Industries for BA27 and BC05, Dr. Toshiharu Suzuki (Hokkaido University) for UT18, and Naoki Sawano for technical assistance.

Correspondence should be addressed to Dr. Yasuo Ihara, Department of Neuropathology, Faculty of Life and Medical Sciences, Doshisha University, 4-1-1, Kizugawadai, Kizugawa 619-0225, Japan. E-mail: yihara@mail. doshisha.ac.jp.

DOI:10.1523/JNEUROSCI.2362-09.2009

Copyright $\odot 2009$ Society for Neuroscience $\quad$ 0270-6474/09/2913042-11\$15.00/0 cies of longer $A \beta$ s, including $A \beta_{48}, A \beta_{46}, A \beta_{45}$, and $A \beta_{43}$ in the cell lysate (Qi-Takahara et al., 2005). We also found that treatment of cells with $\mathrm{N}$-[N-(3,5-difluorophenacetyl)-L-alanyl]-Sphenylglycine $t$-butyl ester (DAPT) causes intracellular accumulation of longer $\mathrm{A} \beta$ species together with depletion of $\mathrm{A} \beta_{40 / 42}$ in the culture medium. Intracellular depletion of $\mathrm{A} \beta_{40}$ accompanied the accumulation of $\mathrm{A} \beta_{43}$ at low dosage and of $\mathrm{A} \beta_{46}$ at higher dosage (Qi-Takahara et al., 2005). This accumulation profile suggests that the flow of product from $A \beta_{46}$ to $A \beta_{43}$ to $A \beta_{40}$ is prevented downstream, which would cause differential accumulation of first $\mathrm{A} \beta_{43}$ and then $\mathrm{A} \beta_{46}$. This suggested to us that three residues along the $\alpha$-helix of the transmembrane domain (TMD) of $\beta$ CTF would be removed successively to generate $\mathrm{A} \beta_{40}$ (Qi-Takahara et al., 2005).

If the view that longer $A \beta$ s are processed to $A \beta_{40 / 42}$ is correct, longer $\mathrm{A} \beta$ species $\left(\mathrm{A} \beta_{48}-\mathrm{A} \beta_{52}\right)$ should generate $\mathrm{A} \beta_{40 / 42}$ within the cell. Using a transfection experiment, we noted an unusual rule: $\mathrm{A} \beta_{51}$ and $\mathrm{A} \beta_{48}$ preferentially generated $\mathrm{A} \beta_{42}$, whereas other longer $A \beta s$, including $A \beta_{49}, A \beta_{50}$, and $A \beta_{52}$, produced predominantly A $\beta_{40}$ (Funamoto et al., 2004). Although the exact mechanism remains unknown, these results suggest that this three-residue rule is involved in the preference for the final product, $\mathrm{A} \beta_{40}$ or $\mathrm{A} \beta_{42}$. Conversely, DAPT causes accumulation of $\mathrm{A} \beta_{45}$ and $\mathrm{A} \beta_{48}$ in the CHO cells expressing mutant (mt) PS2 (N141I) and mtPS1 (M233T), respectively (Yagishita et al., 2006). Based on these results, it is reasonable to postulate that two product lines are at work: one produces $\mathrm{A} \beta_{40}$ and the other pro- 
duces $\mathrm{A} \beta_{42}$. These observations led us to the view that this process mediated by $\gamma$-secretase proceeds through the stepwise (successive) cleavage of $\beta$ CTF at every third residue. According to this model, $\mathrm{A} \beta_{40}$ is generated through $\mathrm{A} \beta_{43 / 46}$ from $\mathrm{A} \beta_{49}$, which is generated by $\varepsilon$-cleavage, and $\mathrm{A} \beta_{42}$ is generated through $\mathrm{A} \beta_{45}$ from $\mathrm{A} \beta_{48}$, which is generated by $\varepsilon$-cleavage (Qi-Takahara et al., 2005; Kakuda et al., 2006). The $\varepsilon$-cleaved product, $\mathrm{A} \beta_{49}$ on the $\gamma$-secretase complex, releases ITL, VIV, and IAT and is converted to $\mathrm{A} \beta_{40}$. The other $\varepsilon$-cleaved product, $\mathrm{A} \beta_{48}$, releases VIT and TVI and is converted to $\mathrm{A} \beta_{42}$ (tripeptide hypothesis). We tested this model by using liquid chromatography tandem mass spectrometry (LC-MS/MS) to quantify the specific tripeptides in the reaction mixture of a cell-free $A \beta$-generating system and a 3-[(3-cholamidopropyl)dimethylammonio]-2hydroxyl-1-propanesulfonate (CHAPSO)-reconstituted $\mathrm{A} \beta$ generating system.

\section{Materials and Methods}

Cell culture. CHO cells inducibly expressing $\beta$ CTF (C99 cells) (QiTakahara et al., 2005) were maintained in F-12 nutrient mixture (Invitrogen) containing $10 \%$ fetal calf serum (Invitrogen), penicillin/streptomycin, $250 \mu \mathrm{g} / \mathrm{ml}$ zeocin (Invitrogen), $10 \mu \mathrm{g} / \mathrm{ml}$ blasticidin S (Invitrogen), and 200

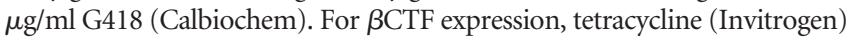
was supplemented into the culture medium at a concentration of $1 \mu \mathrm{g} / \mathrm{ml}$. A $4 \mathrm{~h}$ incubation with tetracycline was found to be suitable for the experiment; longer culture times increased the intensity of an $\alpha \mathrm{CTF}$-like band (data not shown).

Antibodies. The monoclonal antibodies to $\mathrm{A} \beta$ used in this study were 82E1 (highly specific for Asp1 of human A $\beta$ ) (IBL Co. Ltd.), BA27 (raised against $\mathrm{A} \beta_{1-40}$, highly specific for $\mathrm{Val} 40$ ), $\mathrm{BC} 05$ (raised against $\mathrm{A} \beta_{35-43}$, specific for $\mathrm{A} \beta_{42}$ ), 12F4 (specific for $\mathrm{A} \beta_{42}$; Covance), and 9C4 (specific for $\mathrm{A} \beta_{43}$; Covance). A polyclonal antibody UT18 (raised against the 20 residue cytoplasmic domain of APP; a gift from Dr. T. Suzuki, Hokkaido University, Sapporo, Japan) was used to detect $\beta$ CTF and AICD.

Membrane preparation. Confluent cells were harvested and homogenized in homogenization buffer [20 mM piperazine-1,4-bis(2ethanesulfonic acid) (PIPES), pH 7.0, $140 \mathrm{~mm} \mathrm{KCl,} 0.25 \mathrm{~m}$ sucrose, and $5 \mathrm{~mm}$ EGTA]. Homogenized cells were centrifuged at $800 \times g$ to remove nuclei and cell debris. The supernatant was further ultracentrifuged at $100,000 \times g$ for $1 \mathrm{~h}$. The resultant pellet containing the total membrane fraction was suspended in homogenization buffer. The membrane fraction at a protein concentration of $2.5 \mathrm{mg} / \mathrm{ml}$ in homogenization buffer containing protease inhibitor mixture [0.5 mM diisopropylfluorophosphate (DIFP), $1 \mu \mathrm{g} / \mathrm{ml} \mathrm{N}^{\alpha}$-p-tosyl-L-lysyl chloromethyl ketone (TLCK), $10 \mu \mathrm{g} / \mathrm{ml}$ antipain, $10 \mu \mathrm{g} / \mathrm{ml}$ leupeptin, $1 \mathrm{~mm}$ thiorphan, $5 \mathrm{~mm}$ phenanthroline, $100 \mu \mathrm{M}$ bestatin, $10 \mu \mathrm{M}$ amastatin, $0.1 \mu \mathrm{M}$ arphamenine, and 5 mM EDTA] was incubated at $37^{\circ} \mathrm{C}$ for the time indicated, and the reaction was stopped by placing the reaction mixture on ice. After extraction of lipids twice with chloroform/methanol (2:1) and chloroform/methanol/ water (1:2:0.8), the residue was extracted with $70 \%$ formic acid, and the extract was dried. The residue was dissolved in the SDS sample buffer and subjected to $16.5 \%$ SDS-PAGE, followed by Western blotting.

Preparation of C99-FLAG substrate. A C-terminal fragment of APP ( $\beta$ CTF or C99) was fused at the $\mathrm{C}$ terminus with FLAG tag (C99-FLAG) and at the $\mathrm{N}$ terminus with signal peptide (MQLRNPELHLGCALALRFLALVSWDIPGARA) of human $\alpha$-galactosidase A. The resultant coding fragment was inserted into pFASTBAC1 (Invitrogen). Sf9 cells were infected with recombinant baculovirus according to the instructions of the manufacturer (Kakuda et al., 2006). Infected cells (60 ml culture) were harvested after $36 \mathrm{~h}$ and resuspended in $0.3-0.5 \mathrm{ml}$ of Tris-buffered saline ( $50 \mathrm{~mm}$ Tris $\mathrm{HCl}, \mathrm{pH} 7.6$, and $150 \mathrm{~mm} \mathrm{NaCl}$ ). The suspension was mixed with equal amount of $2 \times$ YI buffer [ $50 \mathrm{~mm}$ Tris $\mathrm{HCl}$, pH 7.6, 150 $\mathrm{mm} \mathrm{NaCl}, 2 \% \mathrm{NP}-40$, and $2 \times$ protease inhibitor cocktail (Roche Diagnostics)] and incubated on ice for $1 \mathrm{~h}$. After ultracentrifugation at $245,000 \times g$ for $20 \mathrm{~min}$, the supernatant was agitated with $0.2 \mathrm{ml}$ of anti-FLAG M2 agarose beads (Sigma) overnight. C99-FLAG was eluted from the beads by incubation with $0.2 \mathrm{ml}$ of $100 \mathrm{~mm}$ glycine $\mathrm{HCl}, \mathrm{pH} 2.7$, for $10 \mathrm{~min}$ at room temperature, and the eluate was neutralized immediately by addition of $1 / 25$ volume of $1 \mathrm{M}$ Tris $\mathrm{HCl}, \mathrm{pH}$ 8.0. The concentration of residual NP-40 in the purified C99-FLAG was estimated to be $0.3-0.4 \%$. The eluted C99-FLAG was confirmed for its purity and quantified by Coomassie Brilliant Blue staining after gel electrophoresis (Kakuda et al., 2006).

Immunoprecipitation of the $\gamma$-secretase complex. The membrane fraction from CHO cells was adjusted to a protein concentration of $10 \mathrm{mg} / \mathrm{ml}$ with suspension buffer ( $50 \mathrm{~mm}$ PIPES, pH 7.0, $0.25 \mathrm{~m}$ sucrose, and $1 \mathrm{~mm}$ EGTA). Equal volume of $2 \times$ NK buffer (50 mм PIPES, pH 7.0, $0.25 \mathrm{~m}$ sucrose, $1 \mathrm{~mm}$ EGTA, 2\% CHAPSO, $1 \mathrm{~mm}$ DIFP, $2 \mu \mathrm{g} / \mathrm{ml}$ TLCK, 20 $\mu \mathrm{g} / \mathrm{ml}$ antipain, $20 \mu \mathrm{g} / \mathrm{ml}$ leupeptin, and $2 \mathrm{~mm}$ thiorphan) was added, and the fraction was kept on ice for $1 \mathrm{~h}$. After centrifugation at 100,000 $\times$ $g$ for $1 \mathrm{~h}$, the supernatant was saved as $1 \%$ CHAPSO-solubilized membrane fraction and kept at $-80^{\circ} \mathrm{C}$ until use. The solubilized fraction was diluted in three volumes of CHAPSO-free buffer (50 mM PIPES, pH 7.0, $0.25 \mathrm{~m}$ sucrose, $1 \mathrm{~mm}$ EGTA, $0.5 \mathrm{~mm}$ DIFP, $1 \mu \mathrm{g} / \mathrm{ml}$ TLCK, $10 \mu \mathrm{g} / \mathrm{ml}$ antipain, $10 \mu \mathrm{g} / \mathrm{ml}$ leupeptin, $1 \mathrm{~mm}$ thiorphan, $100 \mu \mathrm{M}$ bestatin, $10 \mu \mathrm{M}$ amastatin, $0.1 \mu \mathrm{M}$ arphamenine, and $5 \mathrm{~mm}$ EDTA). Anti-nicastrin antibody (Sigma) and protein A-Sepharose (GE Healthcare) were added to the CHAPSO-solubilized supernatant, and the supernatant was stirred at $4^{\circ} \mathrm{C}$ overnight. The suspension was centrifuged, and the resultant pellet was washed sufficiently with $0.25 \%$ CHAPSO buffer ( 50 mM PIPES, $\mathrm{pH}$ 7.0, $0.25 \mathrm{~m}$ sucrose, $1 \mathrm{~mm}$ EGTA, and $0.25 \%$ CHAPSO). The $\gamma$-secretase complex bound to protein A-Sepharose was allowed to react with $250 \mathrm{nM}$ C99-FLAG in $0.325 \%$ CHAPSO buffer containing $0.1 \%$ phosphatidylcholine and $1 \mathrm{~mm}$ thiorphan with gentle agitation at $37^{\circ} \mathrm{C}$ for the time indicated (Osawa et al., 2008).

For the time lag (induction period) experiment, particular caution was taken to maintain the temperature of the reaction mixture at $37^{\circ} \mathrm{C}$. Approximately $225 \mu \mathrm{l}$ of $0.325 \%$ CHAPSO buffer containing $0.1 \%$ phosphatidylcholine was kept at this temperature. The protein A-Sepharosebound $\gamma$-secretase $(\sim 20 \mu \mathrm{l})$ that had been kept on ice was mixed with the preincubated CHAPSO buffer and incubated at $37^{\circ} \mathrm{C}$ for $5 \mathrm{~min}$. The reaction was started by adding the substrate, C99-FLAG $(\sim 5 \mu \mathrm{l})$ that had been kept on ice, to the preincubated mixture $(\sim 245 \mu \mathrm{l})$, and the reaction mixture was agitated by inversion in a chamber kept at $37^{\circ} \mathrm{C}$.

A part of the reaction mixtures was treated with TCA at the times indicated, and the remaining part was subjected to quantitative Western blotting for $A \beta$ species using $A \beta$ end-specific antibodies. The reaction mixtures were also separated in a two-layer separating gel system $(20 \%$ acrylamide layer of $5 \mathrm{~cm}$ length and $10 \%$ acrylamide layer containing $8 \mathrm{M}$ urea of $10 \mathrm{~cm}$ length) to visualize smaller $\mathrm{A} \beta$ species, such as $\mathrm{A} \beta_{37}, \mathrm{~A} \beta_{38}$, and $\mathrm{A} \beta_{39}$ (see Figs. $4 A$, right; $7 B$, middle and bottom). Alternatively, the mixtures were loaded on another two-layer separating gel (10\% acrylamide upper layer of $4 \mathrm{~cm}$ length and $10 \%$ acrylamide lower layer containing $8 \mathrm{~m}$ urea of $15 \mathrm{~cm}$ length) together with SeeBlue prestained standard (Invitrogen). Just before the samples ran into the $8 \mathrm{~m}$ urea layer, the gel plate was disassembled, and the upper separating gel containing proteins of $>7 \mathrm{kDa}$ and the stacking gel were removed. The lower separating gel was reassembled and again connected to a power supply for additional separation of $A \beta$ species. This allowed us to distinguish smaller $A \beta$ species from the truncated C99-FLAG substrate with high resolution (see Fig. $4 A$, right). The blots were developed by an enhanced chemiluminescence system (GE Healthcare), and the intensity of the signals was quantified using an LAS-4000 IR multicolor luminescent image analyzer (Fujifilm). The TCA-treated reaction mixture was homogenized and kept on ice for $15 \mathrm{~min}$. The suspension was centrifuged at $204,000 \times g$, and the supernatant was filtrated through a nitrocellulose membrane filter with $0.22 \mu \mathrm{m}$ pore size (Millipore Corporation). The filtrate was injected into a HP1100 (Hewlett-Packard) equipped with CAPCELPAK C18 $(4.6 \times 250 \mathrm{~mm}$; Shiseido $)$ for concentration. The column was washed with $5 \% \mathrm{~B}$ for $20 \mathrm{~min}$, and the peptides were eluted as a mixture by using a steep $5-90 \% \mathrm{~B}$ gradient for $1 \mathrm{~min}$ [A: $0.09 \%$ trifluoroacetic acid (TFA); B: $0.075 \%$ TFA containing $80 \%$ acetonitrile].

LC-MS/MS identification and quantification of tripeptides and other oligopeptides. Quattro Premier XE tandem quadrupole mass spectrometer accompanied by ultra performance liquid chromatography (Waters) was used to identify and quantify the tripeptides and other oligopeptides. 
To quantify each analyte, a combination of precursor ion product ion pair was monitored by multiple-reaction monitoring mode.

The conditions for quantification were established first for the five tripeptides ITL, VIV, IAT, VIT, and TVI, which were assumed to be liberated during incubation. Precursor and product ions to identify and quantify each tripeptide were assigned, and product ions were selected according to the signal intensity: massto-charge ratio $(\mathrm{m} / \mathrm{z})$ of 303.7 and 184.8 for IAT; 329.8 and 184.9 for VIV; 345.8 and 214.9 for ITL; 331.8 and 172.9 for TVI; and 331.8 and 185.0 for VIT. For Phe Leu Phe (FLF) (internal standard), the corresponding values were 425.7 and 119.1. When subjected to the column (AtlantisT3C18, $2.0 \times 150 \mathrm{~mm}$ ), all of these peptides were eluted with a 20 min linear gradient of $0-30 \%$ acetonitrile. A standard curve for each tripeptide was found to be linear from 0.4 to $100 \mathrm{fmol} . R^{2}=0.997$ for IAT, 0.996 for VIV, 0.999 for ITL, 0.998 for TVI, and 0.994 for VIT (supplemental Table S1, available at www.jneurosci.org as supplemental material).

The reaction mixture contained high concentrations of protease inhibitors $(0.5 \mathrm{~mm}$ DIFP, $1 \mu \mathrm{g} / \mathrm{ml}$ TLCK, $10 \mu \mathrm{g} / \mathrm{ml}$ antipain, 10 $\mu \mathrm{g} / \mathrm{ml}$ leupeptin, $1 \mathrm{~mm}$ thiorphan, $100 \mu \mathrm{M}$ bestatin, $10 \mu \mathrm{M}$ amastatin, $0.1 \mu \mathrm{M}$ arphamenine, and $5 \mathrm{~mm}$ EDTA), which may affect the identification and quantification of tripeptides even by LC-MS/MS. Whereas significant proportions of each tripeptide injected alone into HPLC can be recovered in the presence of the above inhibitors, the yield decreased markedly. The reason appeared to be unusual enhancement (as much as fivefold) of the internal standard (FLF), and thus to construct the standard curve for each peptide, protease inhibitors and FLF were always added. It is impossible to assess accurate recoveries from the cell-free system because of the presence of the membrane, which brings the substrate. The measures obtained for the tripeptides released shown in Figure 2, $B$ and $C$, do not account for the loss during incubation and are probably underestimations.

In the experiments using the cell-free system, for unknown reasons, subtle differences in the elution position in LC were observed when compared with the authentic (synthetic) tripeptide. In addition, for unknown reasons, a few to several peaks for one product ion were observed, although the signal should have been double that selected (supplemental Fig. S1, available at www.jneurosci.org as supplemental material). To determine which was the peak of interest, we investigated (1) which peak intensity increased when each synthetic (authentic) peptide was added to the reaction mixture and (2) what the ratio of the intensities of the two selected product ions one precursor ion showed. Based on these two criteria, the peak of interest was identified and quantified (supplemental Fig. S1, available at www.jneurosci.org as supplemental material).

For the peptides examined here, the following precursor and product $\mathrm{m} / \mathrm{z}$ values were used for identification and quantification: 274.0 and 129.0 for GVV; 330.0 and 199.0 for VVI; 302.1 and 213.1 for VIA; 290.0 and 173.0 for ATV; 344.1 and 213.1 for IVI; 332.0 and 187.1 for TLV; and 362.0 and 213.0 for LVM. The following $\mathrm{m} / \mathrm{z}$ values were also selected: 331.1 and 213.9 for GGVV; 401.2 and 170.8 for VVIA; 615.2 and 185.0 for IATVIV; and 657.3 and 184.9 for VIVITL. All the synthetic peptides examined here were ready to be soluble in water and $10 \%$ TCA.

In the experiment using the CHAPSO-reconstituted system, there were almost no extra peaks in the total ion chromatogram that had unknown origins, as seen in the cell-free system, and identifying the peak was straightforward (supplemental Fig. S2, available at www.jneurosci. org as supplemental material). It is likely that the membrane fraction is a very crude source of $\gamma$-secretase and contains many proteases and membrane-associated proteins and their degraded products.

Recoveries. For quantitative comparison between Western blotting and LC-MS/MS, recoveries of the tripeptides, tetrapeptides, and hexapeptides and $\mathrm{A} \beta_{40}, \mathrm{~A} \beta_{42}$, and $\mathrm{A} \beta_{43}$ were measured. Eppendorf-type tubes (1.5 ml, non-stick, RNase-free microfuge tube; Ambion) were used throughout the experiment. Four hundred fifty, 2250, and $4500 \mathrm{fmol}$ of each tripeptide, tetrapeptide, and hexapeptide were added to the tube containing the same reaction mixture without the substrate $(250 \mu \mathrm{l})$, which were kept at $37^{\circ} \mathrm{C}$ for 0 and $1 \mathrm{~h}$. The incubation was terminated by adding TCA to the mixture (the final concentration, 10\%). Resultant TCA supernatants were injected to HPLC (HP1100; Hewlett-Packard) for concentration, and the eluate was dried in a speed vac centrifuge. The solubilized peptides were subjected to LC-MS/MS (Waters). Each fractionated peptide was quantified using each authentic peptide as a standard. Recoveries from non-incubated tubes were similar in the yields with $51.1-58.9 \%$ for all the tripeptides, tetrapeptides, and hexapeptides except VIVITL, which had unusually high recoveries (81.5\%) (supplemental Table S2, available at www.jneurosci.org as supplemental material). Those from incubated tubes were also similar in the yields providing $46.7-54.3 \%$ for all the peptides except VIVITL, which was recovered by $73.1 \%$ (supplemental Table S2, available at www.jneurosci. org as supplemental material). After $1 \mathrm{~h}$ incubation, recoveries of the peptides invariably decreased, and there was no significant difference among the recoveries from 450, 2250, and $4500 \mathrm{fmol}$ peptide/250 $\mu \mathrm{l}$. These decreases may be attributable to nonspecific binding to the tube and/or hydrolytic degradation of the peptides.

Appropriate amounts of $\mathrm{A} \beta_{40}, \mathrm{~A} \beta_{42}$, and $\mathrm{A} \beta_{43}$ that were solubilized in DMSO and kept at $-80^{\circ} \mathrm{C}$ were added to the tubes containing the same reaction mixture without the substrate. The tubes containing final concentrations, 4,8 , or $16 \mathrm{pmol} / \mathrm{ml}$ for each $\mathrm{A} \beta$, were kept at $37^{\circ} \mathrm{C}$ for 0 and 
$\mathbf{A}_{1}$

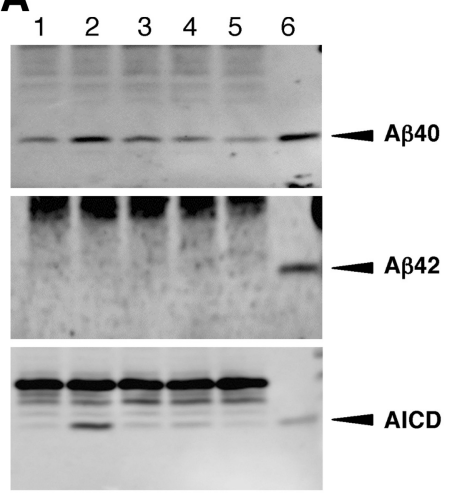

B

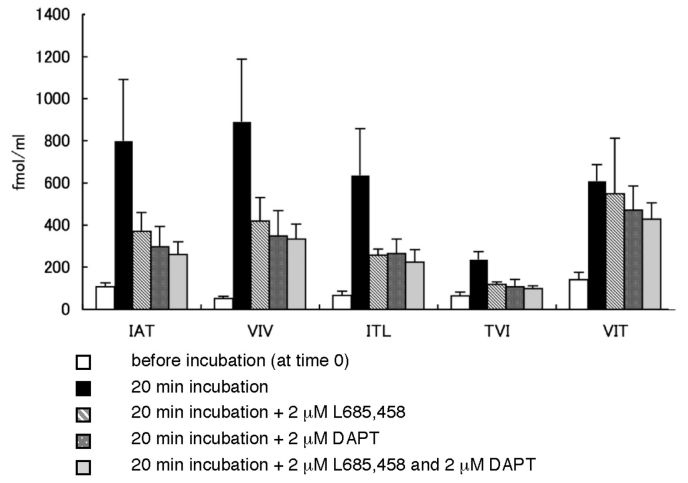

C

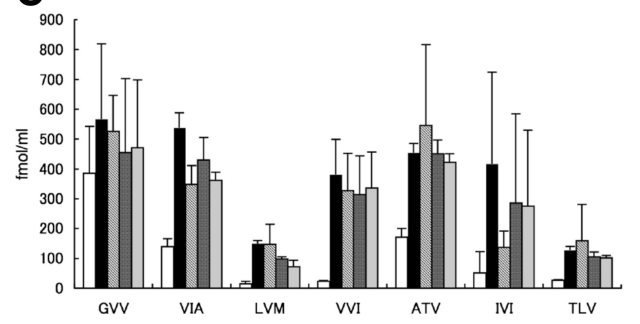

before incubation (at time 0)
20 min incubation
20 min incubation + $2 \mu \mathrm{M}$ L685,458
20 min incubation + $2 \mu \mathrm{M}$ D APT

․ 20 min incubation $+2 \mu \mathrm{M}$ DAPT
$\square \quad 20 \mathrm{~min}$ incubation $+2 \mu \mathrm{M} \mathrm{L685,458}$ and $2 \mu \mathrm{M} \mathrm{DAPT}$

Figure 2. The tripeptides quantified by LC-MS/MS in the reaction mixture of the cell-free A $\beta$-generating system. $A$, The cell-free reaction mixtures before and after incubation were delipidated (see Materials and Methods) and subjected to Western blotting using BA27 (specific for $A \beta_{40}$ ), BC05 (specific for $A \beta_{42}$ ), and UT18 (to detect AICD) (arrowheads). Small amounts of AICD and especially $A \beta_{40}$ are associated with the membranes even at time 0 (lane 1 ), although incubation increased their levels markedly (lane 2). In contrast, in the preparation, $A \beta_{42}$ was barely detectable at time 0 and even after incubation. There were no additional increases in $A \beta_{40}$ and AICD in lanes 3 (in the presence of $2 \mu \mathrm{mL685,458),} 4$ (in the presence of $2 \mu \mathrm{m} \mathrm{DAPT}$ ), and 5 (in the presence of both). Authentic $A \beta_{40}, A \beta_{42}$, and AICD50-99 (6.3 fmol for each) were loaded in lane $6 . B$, The five candidate tripeptides predicted to be released were quantified by LC-MS/MS in the cell-free system. Note the following: (1) the significant background noises at time 0 ; (2) remarkable increases by 20 min incubation in the amounts of tripeptides released; and (3) $>50 \%$ suppression by $\gamma$-secretase inhibitors ( $2 \mu \mathrm{m}$ L685,458 and $2 \mu \mathrm{m}$ DAPT) of IAT, VIV, ITL, and TVI levels. VIT release appeared not to be suppressed significantly by the inhibitors. Data are expressed as the means $\pm S D(n=3)$. Corrections for recoveries were not done (see Materials and Methods). C, Another seven tripeptides aligned on the TMD (from position 39 to position 50 according to A $\beta$ numbering) of $\beta$ CTF were quantified similarly by LC-MS/MS in the reaction mixture of the cell-free system. Note the following: (1) certain tripeptides gave higher signals at time 0 ; (2) large increases in their levels by incubation; and (3) no apparent effects on their release by $\gamma$-secretase inhibitors. Altogether, the results from the cell-free system may suggest that the tripeptide hypothesis is correct, but, to understand better the cleavage mechanism, the assay system should not be contaminated by proteases. Error bars indicate SD $(n=3)$. Corrections for recoveries were not done (see Materials and Methods).

$1 \mathrm{~h}$. Aliquots were taken from the incubated mixtures and subjected to quantitative Western blotting using specific monoclonal antibodies. Incubation did not affect the yields of the three $\mathrm{A} \beta \mathrm{s}$, but the yields differed a little from each another: the yield of $\mathrm{A} \beta_{40}$ was the highest at $85.6 \%$, that of $\mathrm{A} \beta_{43}$ was $84.6 \%$, and that of $\mathrm{A} \beta_{42}$ was $80.7 \%$ (supplemental Table S2, available at www.jneurosci.org as supplemental material).

Quantitative Western blotting. Aliquots from the reaction mixture were dissolved in the SDS sample buffer and subjected to $16.5 \%$ SDS-PAGE, followed by Western blotting using the end-specific $\mathrm{A} \beta$ antibodies BA27, 12F4 (Covance), 9C4 (Covance), and UT18. A $\beta_{40}, \mathrm{~A} \beta_{42}, \mathrm{~A} \beta_{43}$, and AICD on the blot were quantified by an LAS-4000 IR multi color luminescent image analyzer (Fujifilm) with defined amounts of each authentic A $\beta$ (Peptide Institute) and AICD-50-99-FLAG (Kakuda et al., 2006) as the standards. The protein amounts of the authentic standards were determined by amino acid analysis (Peptide Institute).

Other methods and reagents. Tripeptides and other oligopeptides were custom made by the Peptide Institute. Their quantities were determined by amino acid analysis (Peptide Institute).

The $\gamma$-secretase inhibitors L685,458 [1S-benzyl-4R-(1S-carbamoyl-2phenylethylcarbamoyl-1S-3-methylbutylcarbamoyl)-2 R-hydroxy-5phenylpentyl] carbamic acid tert-butyl ester] and DAPT were purchased from Calbiochem. Both were solubilized with DMSO and kept $-80^{\circ} \mathrm{C}$ until use. Protein concentrations were determined using the bicinchoninic acid protein assay (Pierce Chemical).
Numerical simulation. Simulations were performed by numerical integration using fourth-order Runge-Kutta method. The reactions simulated were those shown in supplemental Appendix (Eqs. A1-A5) (available at www. jneurosci.org as supplemental material), which are described in the differential Equations B1B16 in supplemental Appendix (available at www. jneurosci.org as supplemental material), in which we assume that only [E] and [C99] have the nonzero initial concentrations.

The parameters were determined using genetic algorithm (GA) to fit the time lines of tripeptide generated, by minimizing the sum of squared differences between all data points in Figure $8 \mathrm{~A}$ and the numerical calculations. The population size for GA is typically 100 , and each individual has a virtual chromosome encoding 16 floating-point real values representing all of the undetermined model system parameters shown in supplemental Table $4 \mathrm{~S}$ (available at www.jneurosci.org as supplemental material). The number of typical iteration steps is 20,000 , and point mutations and crossover mutations of the virtual chromosomes are allowed to occur during optimization. A set of parameters is provided in supplemental Table $4 \mathrm{~S}$ (available at www.jneurosci.org as supplemental material).

\section{Results}

Distinct tripeptides appear to be released from membrane preparations, and their release is suppressed by specific $\gamma$-secretase inhibitors

We sought to identify by LC-MS/MS the particular tripeptides postulated to be released from the TMD of $\beta$ CTF during $\mathrm{A} \beta$ generation (Qi-Takahara et al., 2005) (Fig. 1A). The cell-free $\mathrm{A} \beta$-generating system, in which the membrane fraction was prepared from $\mathrm{CHO}$ cells expressing $\beta$ CTF (C99), was examined at first because we consider it to be similar to the living cell conditions. The tripeptide hypothesis claims (1) that intramembrane cleavage proceeds from $\varepsilon$-cleavage to $\gamma$-cleavage by releasing tripeptides along the $\alpha$-helix of the TMD of $\beta$ CTF (one helical turn is equivalent to 3.6 residues), and (2) that two product lines result: the first starts from $\mathrm{A} \beta_{49}$ (Leu49) through $\mathrm{A} \beta 46$ and $\mathrm{A} \beta_{43}$ to $\mathrm{A} \beta_{40}$ and the second starts from $\mathrm{A} \beta_{48}$ (Thr48) through $\mathrm{A} \beta_{45}$ to $\mathrm{A} \beta_{42}$ (Fig. $1 A$ ). According to this hypothesis, the $\varepsilon$-cleavage primarily determines the preference for the final $\gamma$-cleavage products. Under normal conditions, $\varepsilon$-cleavage occurs mostly at the carboxyl side of Leu 49 and would occur occasionally at the carboxyl side of Thr48, thus resulting in predominantly $\mathrm{A} \beta_{40}$ with a smaller proportion (10-20\%) of $\mathrm{A} \beta_{42}$ in the cell-free $\mathrm{A} \beta$-generating system (Sato et al., 2003).

Before the LC-MS/MS quantification of the tripeptides, several conditions were set. The mass chromatogram of the five candidate tripeptides and FLF, an internal standard, is shown in Figure $1 B$. Each $12.5 \mathrm{fmol}$ of synthetic IAT, VIV, ITL, TVI, and VIT and $100 \mathrm{fmol}$ of FLF were injected. Each peptide eluted at a distinct time in the LC-MS/MS [ultra performance liquid chromatography accompanied by Quattro Premier XE (Waters)]. A linear standard curve was obtained between 0.4 and $100 \mathrm{fmol}$ for 
each tripeptide examined (Fig. 1C) (supplemental Table S1, available at www.jneurosci. org as supplemental material). The enzymatic reaction was terminated with TCA (final concentration, 10\%), and the acid supernatant was subjected to HPLC (HP1100; Hewlett-Packard) for concentration. The concentrates were subjected to LC-MS/MS, and each peptide was quantified using synthetic tripeptides as standards.

When the membrane preparation is incubated at $37^{\circ} \mathrm{C}, \mathrm{A} \beta$ and $\mathrm{AICD}$ are generated and their production is blocked by specific $\gamma$-secretase inhibitors (Sato et al., 2003) (Fig. 2A). The production rates for both molecules were similar (Fig. 2A) and constant up to $10 \mathrm{~min}$ and then declined (Sato et al., 2003). As shown in Figure $2 B$, the amounts of the five tripeptides released increased markedly after 20 min incubation. To confirm that these increases were mediated by $\gamma$-secretase, two inhibitors, L685,458 (transition-state analog) or DAPT (non-transition-state analog), were added. Either inhibitor suppressed the production of IAT, VIV, ITL, and TVI $>50 \%$ but little that of VIT (Fig. $2 B$ ). Thus, the cell-free $\mathrm{A} \beta$ generating system appeared to generate the candidate tripeptides (Fig. $2 B$ ) as well as $A \beta$ and AICD. To examine whether only these five tripeptides are released in the cell-free system, another seven randomly selected tripeptides aligned on the TMD sequence of $\beta$ CTF (GVV, VIA, LVM, VVI, ATV, IVI, and TLV) (Fig. $1 A$ ) were quantified similarly. Among these, ATV, IVI, and TLV together represent a possible $\mathrm{A} \beta_{41}$-producing process (Fig. $1 A)$. Although the production of these seven tripeptides increased substantially after incubation, the amount produced was less than that of the five postulated tripeptides and was not suppressed significantly by L685,458 or DAPT (Fig. 2C). This suggests that these seven tripeptides are produced primarily by other protease(s), probably those associated with the membrane, and not by $\gamma$-secretase.

\section{The CHAPSO-reconstituted $\gamma$-secretase system generates exclusively the candidate tripeptides, and their release is suppressed completely by specific $\gamma$-secretase inhibitors} The above results from the cell-free system suggest that the generation of the five candidate tripeptides is mediated by $\gamma$-secretase, an observation that may support the tripeptide hypothesis. However, significant ambiguity exists, possibly because of contamination of the $\gamma$-secretase system by active proteases. We were surprised by the findings that large amounts of tripeptides were generated, probably from membrane-associated proteins other than $\beta$ CTF, and that this
B
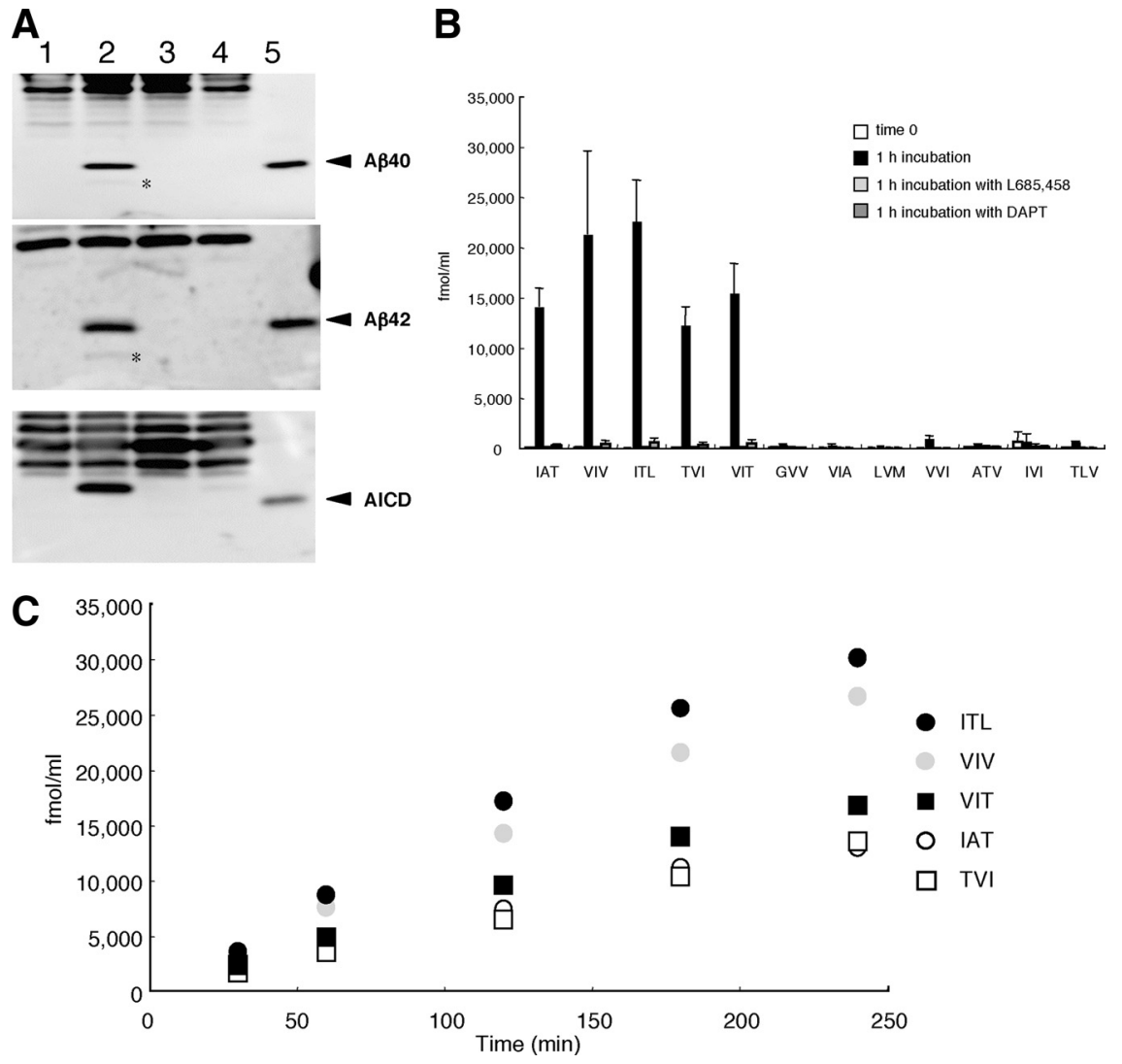

Figure 3. LC-MS/MS quantification of the tripeptides released in the CHAPS0-reconstituted $\gamma$-secretase system. $A$, The reaction mixture was sampled at time 0 (leftmost lane) and $1 \mathrm{~h}$ (the next three lanes) and subjected to SDS-PAGE followed by Western incubation; lane 3, $1 \mathrm{~h}$ incubation in the presence of $2 \mu \mathrm{m} \mathrm{L685,458;} \mathrm{lane} \mathrm{4,} 1 \mathrm{~h}$ incubation in the presence of $2 \mu \mathrm{m}$ DAPT; lane 5 , (to $A \beta_{40}$ (top), $A \beta_{42}$ (middle), and AICD50-99 (bottom) with 18.8 fmol for each. Barely discernible bands (*) below the $A \beta_{40}$ or $A \beta_{42}$ band presumably represent $\mathrm{p} 3\left(\mathrm{~A} \beta_{17-40}\right.$ and $A \beta_{17-42}$; top and middle). AICD in lane 2 (bottom) migrates slower than CHAPSO-reconstituted $\gamma$-secretase system by $1 \mathrm{~h}$ incubation. Note the following: (1) the levels of released tripeptides in the reconstituted system were approximately fivefold higher than in the cell-free system; (2) background noises were very low at time (column 1 for each tripeptide); (3) release of all of the predicted tripeptides was increased markedly by incubation (column 2); (4) hese five tripeptides, the levels of the other seven tripeptides were almost negligible ( $1 / 50$ to $1 / 100$ the values of the 5 tripeptides). IVI, and $48.7 \%$ for TLV (see Materials and Methods) (supplemental Table S2, available at www.jneurosci.org as supplementa reactions proceeded linearly up to $\sim 1 \mathrm{~h}$ and then declined gradually. The relationships ITL $>$ VIV $>$ IAT and VIT $>$ TVI were maintained throughout. Corrections for the recoveries were the same as above. Black circles, ITL; gray circles, VIV; white circles, IAT; black squares, VIT; white squares, TVI.

was insensitive to $\gamma$-secretase inhibitors. Thus, we chose the CHAPSO-reconstituted $\gamma$-secretase system with minor modification developed previously in our laboratory (Kakuda et al., 2006; Osawa et al., 2008). This comprises immunoprecipitated CHAPSO-solubilized $\gamma$-secretase and $\beta$ CTF (C99-FLAG) purified from infected Sf9 cells. Incubating the CHAPSO-containing reaction mixture increased the levels of $A \beta_{49}, A \beta_{48}, A \beta_{45}, A \beta_{43}$, $\mathrm{A} \beta_{42}$, and $\mathrm{A} \beta_{40}$ linearly up to $\sim 2 \mathrm{~h}$, as judged by $\mathrm{A} \beta$ Western blotting (Kakuda et al., 2006) (Fig. 3A). Probably because of the lipidated substrate, the specific activity of $\gamma$-secretase in the reconstituted system was 20 times higher than that reported (Kakuda et al., 2006). The CHAPSO-reconstituted system is characterized by production of abundant $A \beta_{40}, A \beta_{42}$, and $A \beta_{43}$ and small or trace amounts of $\mathrm{A} \beta_{45}, \mathrm{~A} \beta_{48}$, and $\mathrm{A} \beta_{49}$ (Kakuda et al., 2006). In our hands, $A \beta_{46}$ was undetectable by Western blotting 
A

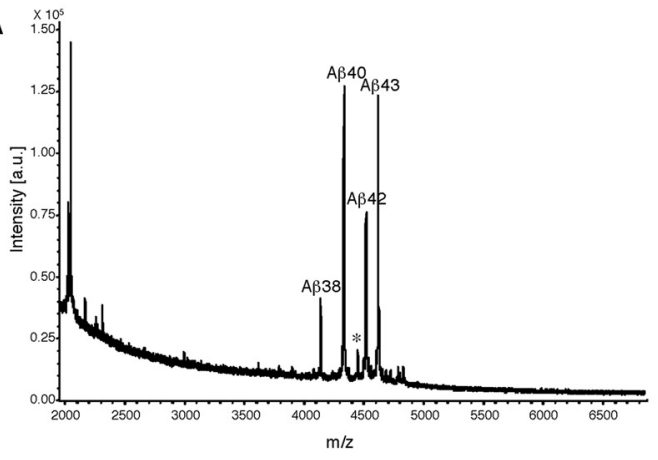

B

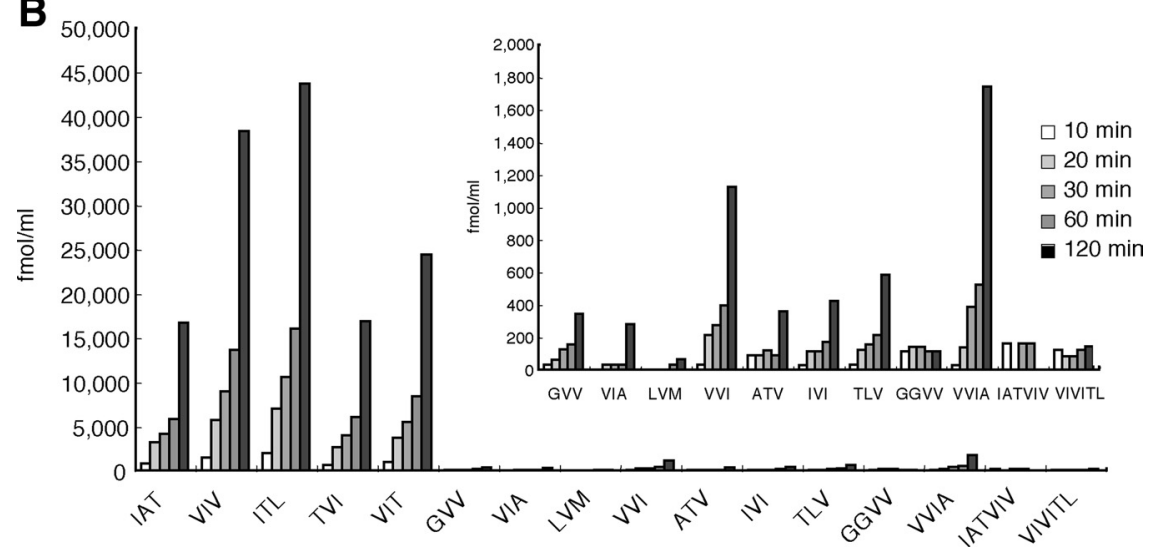

Figure 4. The successive cleavage by the CHAPSO-reconstituted system produces finally $A \beta_{40}$ and $A \beta_{38}$ but not smaller $A \beta$ s. $A$, Left, The reaction mixture after $1 \mathrm{~h}$ incubation was immunoprecipitated with $82 \mathrm{E} 1$, and the precipitate was subjected to TOF mass spectrometry. Four distinct peaks were identified, and their $m / z$ values corresponded to those of $A \beta_{38}, A \beta_{40}, A \beta_{42}$, and $A \beta_{43}$. A small peak indicated by ${ }^{*}$ that is located between the $A \beta_{40}$ and $A \beta_{42}$ peaks may not be $A \beta_{41}$ judging from the observed $\mathrm{m} / \mathrm{z}$. Right, $82 \mathrm{E} 1$ Western blotting after modified urea/SDS-PAGE, which prevented superimposition of $\beta C$ TF on A $\beta$ s so that $A \beta \mathrm{s}$ as small as $A \beta_{37}$ were identified. The reaction mixture after $2 \mathrm{~h}$ incubation contained $A \beta_{45}$ and longer species, $A \beta_{43}, A \beta_{42}, A \beta_{40}$, and $A \beta_{38}$, but not $A \beta_{37}$ (lane 3 ), a finding that was consistent with the results of TOF mass spectrometry. $A \beta_{42 / 43}$ were not well separated under the conditions (lanes 3, 4). Combined authentic $A \beta_{37}-A \beta_{49}$ were loaded in the leftmost and rightmost lanes. $\boldsymbol{B}$, The reaction mixture was sampled at $10 \mathrm{~min}$ to $2 \mathrm{~h}$ and subjected to LC-MS/MS analysis. The levels of the five tripeptides increased markedly in a time-dependent manner. The levels of VVI and VVIA also increased significantly. The levels of other tripeptides, including GVV, VIA, ATV, IVI, and TLV, show very small increases (inset), and the levels of GGVV, IATVIV, and VIVITL did not change after incubation up to $2 \mathrm{~h}$. The recoveries of the 12 tripeptides were the same as above, and those of the tetrapeptides and hexapeptides were assumed to be $52.8 \%$ for GGVV, 54.3\% for VVIA, 46.7\% for IATVIV, and 73.1\% for VIVITL (see the legend to Fig. 3 and supplemental Table 52 , available at www.jneurosci.org as supplemental material).

(Kakuda et al., 2006). As expected, L685,458 uniformly suppressed the production of all A $\beta$ species. Unexpectedly, DAPT did not cause buildups of $\mathrm{A} \beta_{43}$ and $\mathrm{A} \beta_{46}$ but uniformly suppressed the production of all $\mathrm{A} \beta$ species, as did L685,458 (Kakuda et al., 2006).

$\gamma$-Secretase complex immunoprecipitated by anti-nicastrin antibody from the CHAPSO-solubilized membrane fraction was allowed to react with $250 \mathrm{~nm}$ C99-FLAG in $0.325 \%$ CHAPSO buffer containing $0.1 \%$ phosphatidylcholine at $37^{\circ} \mathrm{C}$ (Osawa et al., 2008). The amounts of the five predicted peptides increased greatly during the incubation, but, in sharp contrast, those of the other seven tripeptides no longer increased to such extents in this system (Fig. $3 B$ ). The production of the predicted five tripeptides was suppressed almost completely by $1 \mu \mathrm{M}$ L685,458 or $1 \mu \mathrm{M}$ DAPT (Fig. 3B). This strongly suggests that the five predicted tripeptides are produced specifically by $\gamma$-secretase. These results substantiate the tripeptide hypothesis that particular tripeptides are released exclusively and further support our view that $\mathrm{A} \beta$ generation involves two product lines.
Time course followed by LC-MS/MS indicates that the processing proceeds in a direction from $\boldsymbol{\varepsilon}$ - to $\boldsymbol{\gamma}$-cleavage Because the profile of tripeptides released was consistent with the tripeptide hypothesis (Qi-Takahara et al., 2005), the release kinetics in the CHAPSO-solubilized system was followed by LC-MS/MS. As shown in Figure $3 C$, the levels of all five tripeptides elevated linearly up to $\sim 1 \mathrm{~h}$ and then declined gradually. This suggests that other proteases did not contaminate the reaction mixture to a significant level. This pattern corresponded well with the time course of $A \beta$ produced shown by Western blotting (Kakuda et al., 2006). Importantly, the levels of tripeptides were invariably ITL $>$ VIV $>$ IAT and VIT $>$ TVI from $30 \mathrm{~min}$ to $\sim 4 \mathrm{~h}$. These relationships were maintained strictly up to as early as 10 min (see below).

The tripeptide hypothesis claims that particular tripeptides are released successively to produce $A \beta_{49}, A \beta_{46}, A \beta_{43}$, and $\mathrm{A} \beta_{40}$, and $\mathrm{A} \beta_{48}, \mathrm{~A} \beta_{45}$, and $\mathrm{A} \beta_{42}$ in this order (Qi-Takahara et al., 2005). A critical requirement for this model is that the amounts of tripeptides released earlier are definitely larger than the amounts released later. Thus, for the tripeptide hypothesis to be valid, the relationship ITL $>$ VIV $>$ IAT and VIT $>$ TVI should be observed at any time during the reaction. Notably, this relationship itself indicates strongly that the direction of reaction is from $\varepsilon$ - to $\gamma$-cleavage.

\section{The final products of the CHAPSO-} reconstituted $\gamma$-secretase system are $A \beta_{40}$ and $A \beta_{38}$, the latter of which accompanies the release of tetrapeptide In the cell culture system, smaller $A \beta$ s, including $\mathrm{A} \beta_{39}, \mathrm{~A} \beta_{38}, \mathrm{~A} \beta_{37}, \mathrm{~A} \beta_{35}$, and $\mathrm{A} \beta_{34}$, are found in the medium, and their production seems to be mediated by $\gamma$-secretase because their production is suppressed with a specific $\gamma$-secretase inhibitor (Beher et al., 2002). However, one should be cautious about the interpretation because the data do not exclude the possibility that the culture medium contains carboxyl peptidases with robust activity toward $\mathrm{A} \beta$ s of conventional lengths (for example, $\mathrm{A} \beta_{38}, \mathrm{~A} \beta_{40}$, or $\mathrm{A} \beta_{42}$ ) and thus generates smaller $\mathrm{A} \beta$ s (smaller than $\mathrm{A} \beta_{38}$ ). We examined to what extent the CHAPSOreconstituted system processes $\beta \mathrm{CTF}$ and whether the smaller $\mathrm{A} \beta \mathrm{s}$ as observed often in the culture media are produced. After incubation, the reaction mixture was immunoprecipitated with 82E1 (specific for the $\mathrm{N}$ terminus of human $\mathrm{A} \beta$ ), and the precipitate was subjected to time of flight (TOF) mass spectrometry. Four distinct peaks were observed, and their observed $m / z$ values corresponded to those of $\mathrm{A} \beta_{38}, \mathrm{~A} \beta_{40}, \mathrm{~A} \beta_{42}$, and $\mathrm{A} \beta_{43}$ (Fig. $4 A$, left). Longer $\mathrm{A} \beta$ s, including $\mathrm{A} \beta_{45}, \mathrm{~A} \beta_{48}$, and $\mathrm{A} \beta_{49}$ that exist in the reaction mixture (Kakuda et al., 2006), were undetectable by TOF mass spectrometry, presumably because they existed in only small amounts in the mixture and because their ionization efficiencies are very low. Notably, $A \beta$ s smaller than $A \beta_{38}$ were un- 


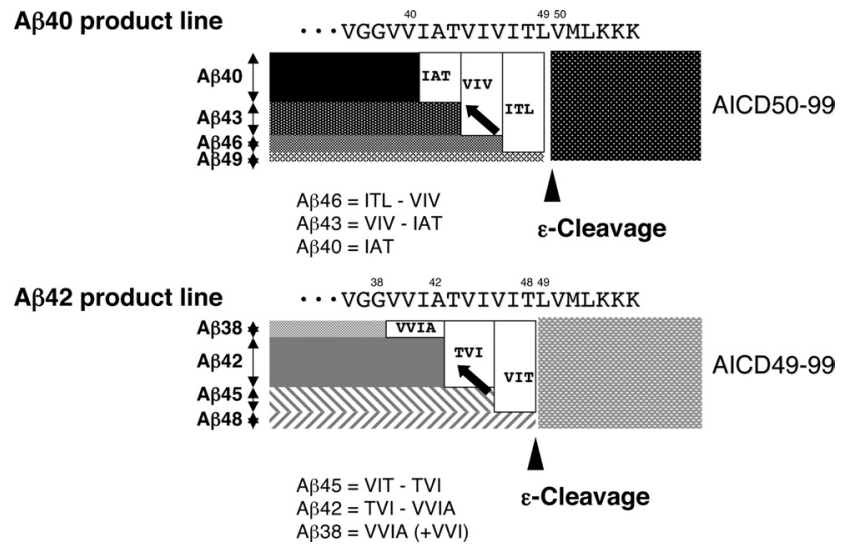

Figure 5. Illustration of the stepwise processing of $\beta C T F . A \beta_{40}$ product line: $A \beta_{49}$ generated by $\varepsilon$-cleavage (arrowhead) is processed successively probably on the $\gamma$-secretase complex. $A \beta_{49}$ is converted to $A \beta_{46}$ by releasing ITL, and $A \beta_{46}$ is converted to $A \beta_{43}$ by releasing VIV. Finally, $A \beta_{43}$ is converted to $A \beta_{40}$ by releasing IAT (arrow). The characteristic of this model is that the difference in the amounts of tripeptide released determines the amount of $A \beta$ produced. Accordingly, $A \beta_{46}=$ ITL - VIV, $A \beta_{43}=$ VIV $-I A T$, and $A \beta_{40}=I A T$. $A \beta_{42}$ product line: $A \beta_{48}$ generated by $\varepsilon$-cleavage (arrowhead) is converted to $A \beta_{45}$, and $A \beta_{45}$ in turn is converted to $A \beta_{42} \cdot A \beta_{42}$ is converted finally to $A \beta_{38}$ (arrow). Similarly, $A \beta_{45}=$ VIT - TVI, $A \beta_{42}=$ TVI - VVIA, and $A \beta_{38}=$ VVIA (see Results). The final step in the $A \beta_{42}$ product line is removal of VVIA, instead of VIA.

detectable by TOF mass spectrometry. In this case, ionization efficiencies should not be low, and thus it is more likely that $\mathrm{A} \beta \mathrm{s}$ smaller than $\mathrm{A} \beta_{38}$ would be absent or at negligible levels in the reaction mixture. To confirm this result, we used a modified urea/SDS-PAGE protocol that makes it possible to identify $A \beta$ s as small as $\mathrm{A} \beta_{37}$. We detected $\mathrm{A} \beta_{38}, \mathrm{~A} \beta_{40}, \mathrm{~A} \beta_{42}$, and $\mathrm{A} \beta_{43}$ (and longer $\mathrm{A} \beta$ species) but not $\mathrm{A} \beta_{37}$ or $\mathrm{A} \beta_{39}$ in the reaction mixture (Fig. $4 A$, right), an observation that is consistent with the TOF mass spectrometry result. We conclude that the CHAPSOreconstituted system proceeds to the production of $\mathrm{A} \beta_{40}$ and $\mathrm{A} \beta_{38}$ but no further.

This raised an important question: is $A \beta_{38}$ produced from $\mathrm{A} \beta_{42}$ by releasing tetrapeptide, VVIA, instead of a tripeptide (QiTakahara et al., 2005)? If so, this would contradict the tripeptide hypothesis. We examined again in depth whether the reaction mixture contains the peptides including GVV, VVI, VIA, and VVIA, which would explain the generation of small A $\beta$ s. Removing GVV from $\mathrm{A} \beta_{40}$ should generate $\mathrm{A} \beta_{37}$, removing VVI from $\mathrm{A} \beta_{41}$ should generate $\mathrm{A} \beta_{38}$, and removing VIA from $\mathrm{A} \beta_{42}$ should generate $\mathrm{A} \beta_{39}$. Among these five peptides, only VVIA was detected consistently in the reaction mixture, although its level was relatively low (Fig. $4 \mathrm{~B}$ ). Thus, the presence of VVIA indicates conversion of $\mathrm{A} \beta_{42}$ to $\mathrm{A} \beta_{38}$ by releasing tetrapeptide, which contradicts the tripeptide rule. Tetrapeptide release is apparently inherent in the $\mathrm{A} \beta_{42}$ processing because the amount of GGVV that may have been released to produce $\mathrm{A} \beta_{36}$ from $\mathrm{A} \beta_{40}$ was unaltered (Fig. $4 B$, inset). Occasionally, VVI was released to a significant level, indicating a very low level of $\mathrm{A} \beta_{41}$ generated in the reaction mixture. This means that $A \beta_{38}$ generation also requires a very small contribution from $\mathrm{A} \beta_{41}$. The failure to detect incubationdependent changes in the levels of two hexapeptides, IATVIV and VIVITL, is noteworthy. This indicates clearly that cleavage of hexapeptide by skipping one helical turn, which still follows the $\alpha$-helical model, never occurred (Fig. 5) (Yagishita et al., 2008).

Closer inspection of Figure $4 B$ shows that very minute amounts ( $1 / 50$ to $1 / 100$ of the candidate tripeptides) of GVV, VIA, ATV, IVI, and TLV were released and that the amount in- creased in a time-dependent manner, which was detectable by LC-MS/MS (Fig. $4 B$, inset). Among the above peptides, ATV, IVI, and TLV were involved in the $\mathrm{A} \beta_{41}$-producing process (Fig. $1 A)$. It is likely that, although very minor, $\mathrm{A} \beta_{41}$ product line starting from $\varepsilon$-cleavage at the carboxyl side of Val-50 also appeared in the CHAPSO-reconstituted system.

\section{Comparison between $\mathrm{A} \boldsymbol{\beta}$ levels determined by Western blotting and those assessed by released tripeptide and} tetrapeptide levels according to the stepwise-processing model According to the stepwise-processing model that was developed from the tripeptide hypothesis (Fig. 5), $\mathrm{A} \beta_{46}=\mathrm{ITL}-\mathrm{VIV} ; \mathrm{A} \beta_{43}=$ VIV - IAT; and $\mathrm{A} \beta_{40}=$ IAT (when additional cleavage does not occur); and $\mathrm{A} \beta_{45}=$ VIT - TVI; $\mathrm{A} \beta_{42}=$ TVI - VVIA; and $\mathrm{A} \beta_{38}=$ VVIA ( + VVI) (when additional cleavage does not occur). The $\mathrm{A} \beta$ quantities inferred from LC-MS/MS according to the model were found to be in parallel with those by Western blotting. In the CHAPSO-reconstituted system, $\mathrm{A} \beta_{40}, \mathrm{~A} \beta_{42}$, and $\mathrm{A} \beta_{43}$ were the most abundant species identified by Western blotting (Fig. 6A). This was also the case with LC-MS/MS quantification (Figs. 5, 6B). Whereas $A \beta_{46}$ was undetectable by Western blotting (Kakuda et al., 2006), LC-MS/MS was able to measure the lowest amounts of possible $\mathrm{A} \beta_{46}$ level (Fig. $3 C$ ).

Figure 6, $A$ and $B$, shows three experiments for the quantitative comparison between Western blotting and LC-MS/MS data. Appropriate amounts of aliquots taken from the reaction mixture after $1 \mathrm{~h}$ were subjected to Western blotting (Fig. 6A) and LC-MS/MS (Fig. $6 B$ ). Instead of urea/SDS-PAGE, conventional SDS-PAGE was used because the former yields broad bands with unclear boundaries, which make it difficult to quantify accurately. To quantify each A $\beta$ species using SDS-PAGE and Western blotting, we used end-specific monoclonal antibodies, BA27, $12 \mathrm{~F} 4$, and $9 \mathrm{C} 4$, that can distinguish between $\mathrm{A} \beta_{40}, \mathrm{~A} \beta_{42}$, and $\mathrm{A} \beta_{43}$, which have the same electrophoretic mobility (Fig. 6A). $\mathrm{A} \beta_{42}$ was also quantified with $\mathrm{BC} 05$. Although $\mathrm{BC} 05$ cross-reacts with $\mathrm{A} \beta_{43}$ (Iwatsubo et al., 1994), the reactivity is $1 / 5$ to $1 / 10$ less than that with $\mathrm{A} \beta_{42}$ in Western blotting. $\mathrm{BC} 05$ and $12 \mathrm{~F} 4$ gave very similar results (data not shown). As shown in Figure $6 B$, the results of the two methods corresponded roughly to each other within at most a twofold difference. Some issues remain to be addressed. Recovery values used here were derived on some assumptions (see Materials and Methods). As described above, the levels of $\mathrm{A} \beta$ s estimated from the levels of tripeptide and tetrapeptide quantified by LC-MS/MS are based on the assumption that there is no leaky pathway other than the two product lines. In view of the completely different methodologies and assumptions for quantification, the results of these methods should appear consistent. Thus, the above results suggest that the stepwiseprocessing model is accurate and that the action of $\gamma$-secretase or the effect of its inhibitors can be considered based on this model.

The above results suggest that the tetrapeptide-release step from $\mathrm{A} \beta_{42}$ to $\mathrm{A} \beta_{38}$ would be a specific target of $\mathrm{A} \beta_{42}$-lowering nonsteroidal anti-inflammatory drugs (NSAIDs) (Weggen et al., 2001; Eriksen et al., 2003) and other $\mathrm{A} \beta_{42}$-enhancing compounds (Kukar et al., 2005). If elucidated, the cleavage mechanism at this step would lead to the development of efficient and selective A $\beta$ modulator. We examined whether sulindac sulfide affects the release of peptides in the CHAPSO-reconstituted system using LC-MS/MS (Fig. 7A) and urea/SDS-PAGE followed by Western blotting (Fig. $7 B$ ). Addition of sulindac sulfide caused a nonsignificant increase in the amount of VVIA in the reaction mixture (Fig. 7A, top), whereas the amount of VVI stayed at the same. The putative levels of $\mathrm{A} \beta_{40}, \mathrm{~A} \beta_{46}$, and $\mathrm{A} \beta_{45}$ did not change sig- 


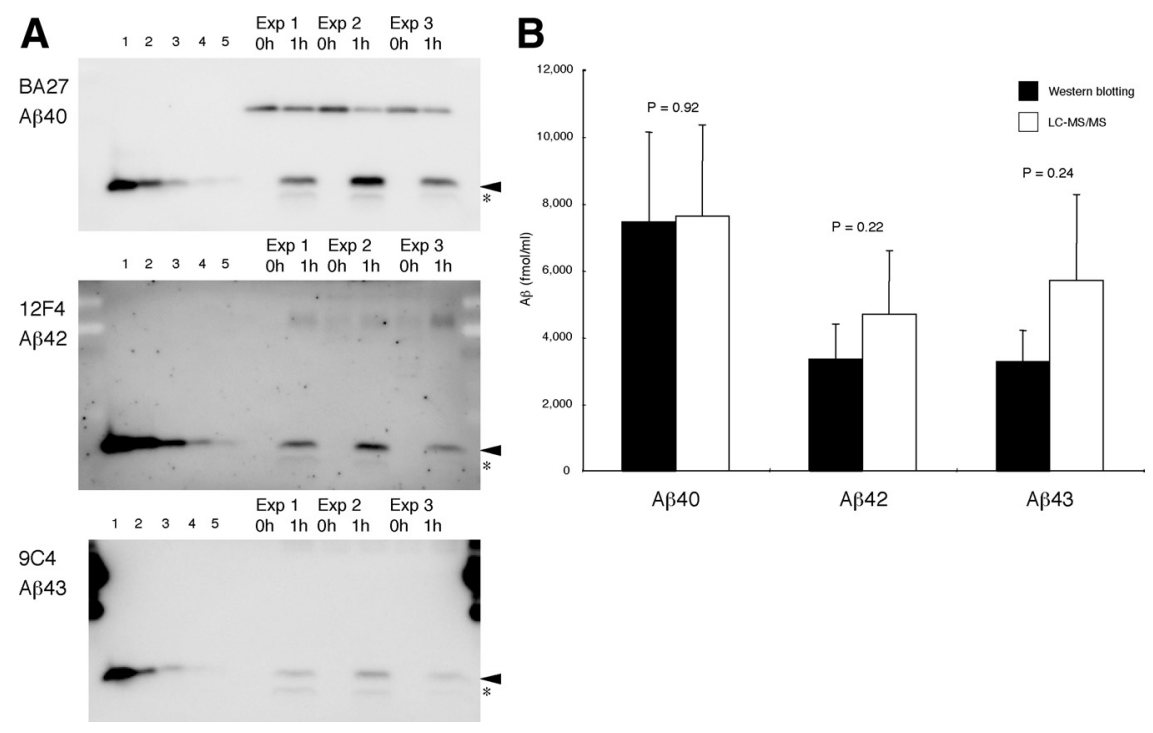

Figure 6. Quantitative comparisons between Western blotting and LC-MS/MS. Three experiments were performed using the same lot of substrate (250 nм C99-FLAG) and the same CHAPSO-solubilized supernatant for immunoprecipitation. One-half of the aliquots taken from the reaction mixture was subjected to conventional PAGE, and the other half was treated with TCA and subjected to LC-MS/MS to quantify the tripeptides. $A$, The left five lanes in each panel were for the standards: $2.5,5,12.5,25$, and $50 \mathrm{fmol}$ of each authentic $A \beta$ was loaded in lanes $1-5$ and subjected to quantitative Western blotting with $B A 27$ for $A \beta_{40}$ (top), with $12 \mathrm{~F} 4$ (middle) for $A \beta_{42}$, and with $9 C 4$ (bottom) for $A \beta_{43}$. The samples before and after $1 \mathrm{~h}$ incubation were loaded in the right six lanes. The reaction mixture generated significant levels of $A \beta_{40}, A \beta_{42}$, or $A \beta_{43}$ after $1 \mathrm{~h}$ incubation (arrowheads). Barely discernible bands indicated by * may represent $\mathrm{p} 3\left(A \beta_{17-40}\right.$ and $\left.A \beta_{17-42}\right)$. $B$, Quantification of generated $A \beta_{40}, A \beta_{42}$, and $A \beta_{43}$ by Western blotting (black column) and by LC-MS/MS based on the stepwise-processing model (white column). Data are expressed as the means $\pm S D(n=3)$. The recoveries for $A \beta$ s were assumed to be $85.6 \%$ for $A \beta_{40}, 80.7 \%$ for $A \beta_{42}$, and $84.6 \%$ for $A \beta_{43}$ (supplemental Table $\mathrm{S} 3$, available at www.jneurosci.org as supplemental material). No significant difference ( $p=0.92$ for $\mathrm{A} \beta_{40}$, 0.22 for $A \beta_{42}$, and 0.24 for $A \beta_{43}, t$ test) between Western blotting and LC-MS/MS data.

nificantly, but the levels of $\mathrm{A} \beta_{43}$ and $\mathrm{A} \beta_{42}$ decreased significantly (Fig. 7A, bottom). TOF mass spectrometry and urea/SDS-PAGE show a characteristic "NSAID effect" pattern in the CHAPSOreconstituted system (Fig. $7 B$ ): a decrease in $A \beta_{42}$ level and an increase in $\mathrm{A} \beta_{38}$ level (for control, see Fig. 4A). A decrease in $\mathrm{A} \beta_{43}$ level has not been reported thus far, possibly because $\mathrm{A} \beta_{43}$ is retained in the cell and is not secreted into the medium, which most commonly serves as the sample for TOF mass spectrometry and Western blotting. Thus, it is possible that, without analysis of the lysates, one could have overlooked changes in the $\mathrm{A} \beta_{43}$ levels caused by sulindac sulfide. It is possible that sulindac sulfide enhances the final steps of both product lines (release of IAT and VVIA) and thereby lowers the levels of $\mathrm{A} \beta_{43}$ and $\mathrm{A} \beta_{42}$, respectively.

LC-MS/MS reveals the presence of an induction period in the generation of tripeptides from $\beta C T F$, which may be explained by the stepwise-processing model

Because the profile of tripeptides released was consistent with the tripeptide hypothesis (Qi-Takahara et al., 2005), the kinetics of the CHAPSO-solubilized system was traced back by LCMS/MS up to as early as 5 min (Fig. $8 A$ ). We thought that accurate quantification of very low amounts of tripeptides, especially in the early phase of the reaction, would provide insight into the cleavage mechanism. We emphasize that the levels of tripeptides were invariably ITL $>$ VIV $>$ IAT and VIT $>$ TVI even at earlier times, as early as $10 \mathrm{~min}$. Most unexpectedly, LC-MS/MS quantification revealed an induction period, 3-4 min, in the generation of peptides. When extrapolated, each time line for each tripeptide, appears to intercept almost the same point on the $x$-axis (Fig. $8 A$ ). After this induction period, the reaction proceeds at a constant rate for each tripeptide.
We are careful about performing this kind of experiment because the $\gamma$-secretase fraction and substrate were kept on ice and then mixed with preincubated CHAPSO buffer. The temperature of the reaction mixture may not reach $37^{\circ} \mathrm{C}$ quickly and thus could be responsible for the induction period when the reaction proceeded very slowly. Despite repeated, very carefully performed, experiments (see Materials and Methods), we still observed the induction period. Thus, the lag cannot be explained by delayed elevation of the mixture temperature to $37^{\circ} \mathrm{C}$. We concluded that the induction period is real in the CHAPSO-reconstituted $\gamma$-secretase system and sought to test whether numerical simulation based on the stepwiseprocessing model can explain this phenomenon. This is because the differential equations (supplemental Appendix, available at www. jneurosci.org as supplemental material) derived from the model do not appear to have analytical solutions.

Here we consider only the $\mathrm{A} \beta_{40}$ product line. When the first-order kinetics is postulated, Equations B1-B16 in supplemental Appendix (available at www.jneurosci.org as supplemental material) can be derived from the stepwise-processing model (Eqs. A1-A5 in supplemental Appendix, available at www.jneurosci.org as supplemental material). These reactions were simulated on the assumption that, in the initial conditions, only [E] and [C99] have the non-zero initial concentrations. The parameters (supplemental Table S4, available at www.jneurosci.org as supplemental material) were fitted to the time lines of tripeptides (Fig. $8 \mathrm{~A}$ ). The simulations showed the following: (1) in the system from as low as $1 \mathrm{fM}$ to as high as $1 \mu \mathrm{M}$ C99 (data not shown), there existed a similar induction period, 3-4 min, for each tripeptide and also a shorter induction period for AICD (Fig. $8 \mathrm{~B}$ ), and then the reactions proceeded linearly up to $6 \mathrm{~h}$ (data not shown; see Fig. 3C); and (2) at $250 \mathrm{~nm} \mathrm{C99} \mathrm{(experimental}$ condition), it takes $10-15 \mathrm{~min}$ for the system to reach the steady state (supplemental Fig. S3, available at www.jneurosci.org as supplemental material). Consistent with these simulation results, we found that an induction period similar to those of tripeptides existed also for AICD, although AICD49-99 and AICD50-99 were not separately measured in the CHAPSO-reconstituted system using quantitative Western blotting (data not shown). Furthermore, in the experiment using $1 \mu \mathrm{M}$ C99, the generation of AICD and tripeptides exhibited induction periods that were very similar to those in the case of 250 nм C99 (data not shown). The simulation showed that the tripeptide generated early has a shorter induction period than one generated late (Fig. $8 B$ ). This may not have been resolved experimentally.

Altogether, the successive reaction kinetics is a reasonable explanation for the induction period in the generation of tripeptides. This suggests strongly that the stepwise-processing model is correct.

\section{Discussion}

Our stepwise-processing model for $\gamma$-secretase cleavage is based on three assumptions: (1) $\varepsilon$-cleavage occurs first and produces $\mathrm{A} \beta_{49}$ and $\mathrm{A} \beta_{48} ;$; (2) the cleavage proceeds from $\varepsilon$-site to $\gamma$-site, and every three-residue and an additional tetrapeptide are removed suc- 

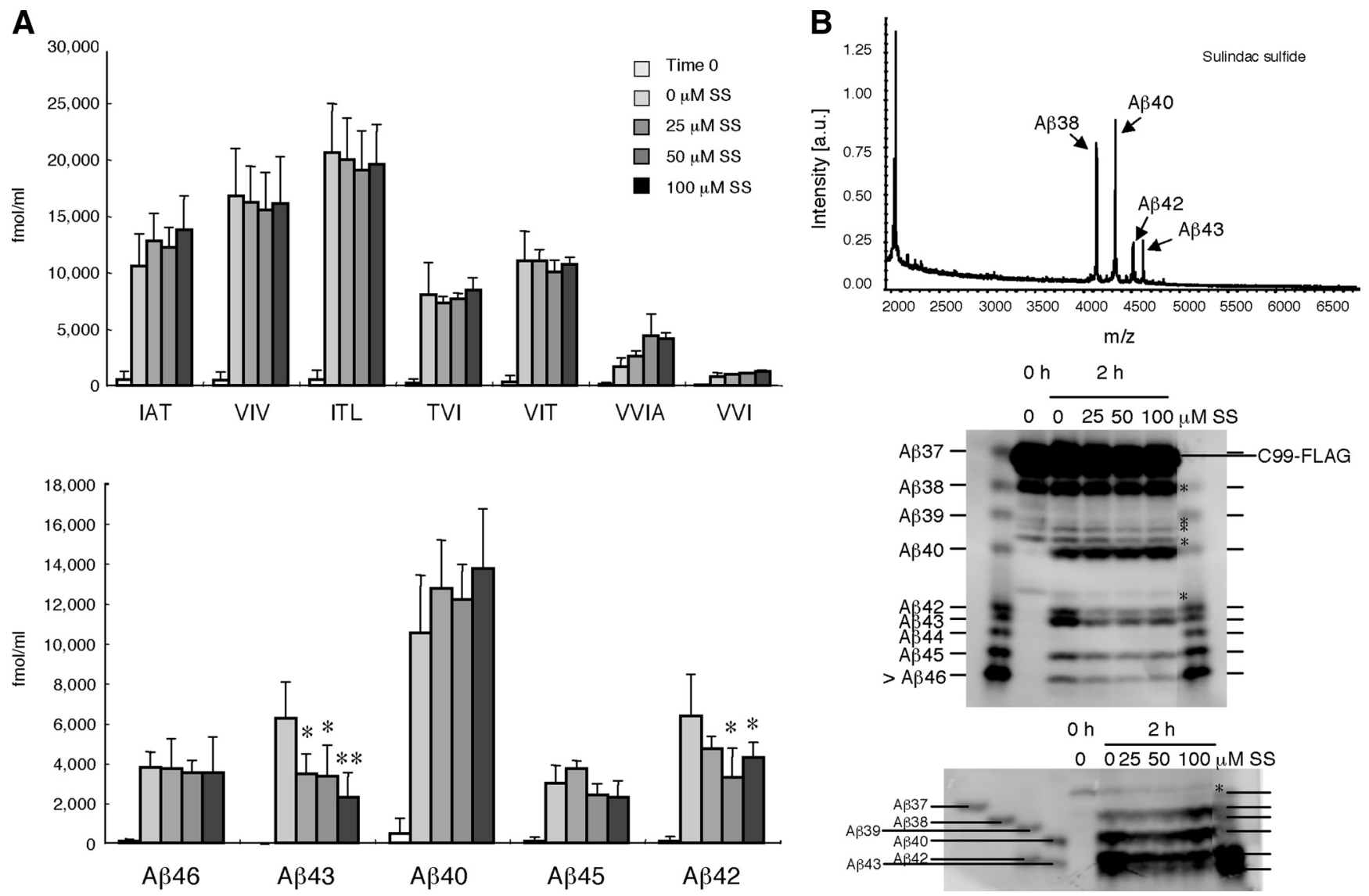

Figure 7. The effects of sulindac sulfide on the stepwise processing. The reaction mixture containing $0,25,50$, or $100 \mu$ m sulindac sulfide was incubated and sampled at $2 \mathrm{~h}$ for LC-MS/MS analysis. $A$, The levels of tripeptides and tetrapeptides release (top) and the calculated levels of A $\beta$ s according to the stepwise-processing model (bottom). Data are expressed as the means \pm SD ( $n=3$ ). The levels of released tripeptides did not change significantly, whereas the level of VVIA tended to increase (although not significantly) with increasing concentrations of sulindac sulfide. Calculated levels of $A \beta_{42}$ and $A \beta_{43}$ were suppressed significantly by sulindac sulfide ( ${ }^{*} p<0.05{ }^{* *} p<0.01$, ANOVA with Bonferroni's post hoc test compared with no treatment). In contrast, the levels of other $A \beta$ s, including $A \beta_{40}, A \beta_{45}$, and $A \beta_{46}$, did not change significantly. The corrections for the peptides were the same as above (see the legends to Figs. 3, 4). $B$, Aliquots from the reaction mixture incubated with or without sulindac sulfide $(100 \mu \mathrm{m}$ ) were immunoprecipitated with $82 \mathrm{E} 1$, and the precipitate was subjected to TOF mass spectrometry (top) and urea/SDS-PAGE [11\% acrylamide gel (middle) and 20/10\% discontinuous acrylamide gel (bottom)], followed by Western blotting with 82E1. TOF mass spectrometry shows that peak heights of $A \beta_{42}$ and $A \beta_{43}$ relative to that of $A \beta_{40}$ decreased compared with those without sulindac sulfide treatment (see Fig. $4 A$ ). The peak height of $A \beta_{38}$ relative to that of $A \beta_{40}$ increased substantially after the treatment (see Fig. $4 A$ ). Western blotting (middle and bottom) showed that (1) $A \beta_{40}$ stayed at a similar level, (2) $A \beta_{42}$ and $A \beta_{43}$ decreased markedly (middle), (3) $A \beta_{38}$ increased (bottom), and (4) $A \beta_{45}$ decreased somewhat (middle). Under the present conditions (middle), $A \beta$ s longer than $A \beta_{45}$ were stuck at the buffer front. ${ }^{*}$ indicates C-terminally truncated C99-FLAG in middle and bottom panels. Overall, the data on the effects of sulindac sulfide based on LC-MS/MS quantification of tripeptides and tetrapeptides corresponded well with those from TOF mass spectrometry and those from quantitative $A \beta$ Western blotting.

cessively from the TMD of $\mathrm{A} \beta_{49 / 48}$; and (3) two predominant product lines are involved: $\mathrm{A} \beta_{49}$ is processed to $\mathrm{A} \beta_{43 / 40}$, and $\mathrm{A} \beta_{48}$ is processed to $\mathrm{A} \beta_{42 / 38}$ (Qi-Takahara et al., 2005). The model implies that, after $\varepsilon$-cleavage, the longer $\mathrm{A} \beta$ s to be processed are kept bound to the $\gamma$-secretase complex until the final processing to $\mathrm{A} \beta_{40 / 38}$; that is, the $k_{-}\left(k_{\mathrm{c}-}, k_{49-}, k_{46-}\right.$, or $\left.k_{43-}\right)$ value for each longer $\mathrm{A} \beta$ is very small (supplemental Appendix, available at www.jneurosci.org as supplemental material). In other words, long $A \beta$ s, including $A \beta_{43}, A \beta_{45}, A \beta_{48}$, and $A \beta_{49}$, detach only rarely from the enzyme complex during the processing (represented by $k_{-}$) because they accumulate gradually in a linear manner in the reaction mixture (Kakuda et al., 2006). Regarding the first point, extensive searching has failed to find such longer AICDs. Although in our present study we did not investigate the temporal profile for the generation of AICD, the presence of an induction period for the generation of tripeptides is consistent with the view that $\varepsilon$-cleavage precedes $\gamma$-cleavage. On the second point, LC-MS/MS showed unambiguously that these postulated tripeptides and tetrapeptides are released into the reaction mixture. Tetrapeptide release is apparently inherent to the $\mathrm{A} \beta_{42}$ pro- cessing, because GGVV, which may have been released to produce $A \beta_{36}$, was undetectable. Relating to the third point, our previous results suggest strongly that the $\mathrm{A} \beta_{40}$-producing process is distinct from the $\mathrm{A} \beta_{42}$-producing process (Funamoto et al., 2004; Qi-Takahara et al., 2005). The latter process was less sensitive to DFK-167, a difluoro ketone peptidomimetic, than was the former. In addition, accumulating $\mathrm{A} \beta_{45}$ in the rafts prepared from N141I mtPS2-transfected cells is not processed to $\mathrm{A} \beta_{42}$, suggesting again that both product lines differ functionally from each other (Yagishita et al., 2008).

The accuracy of LC-MS/MS quantification has also revealed an unexpected cleavage process, which contradicts the threeresidue rule, although it must be a very minor (almost negligible) process. Occasionally, VVI was detected at significant levels, suggesting that a small amount of $\mathrm{A} \beta_{41}$ existed, and thus a small proportion of $\mathrm{A} \beta_{38}$ was derived from $\mathrm{A} \beta_{41}$ in the reaction mixture (Figs. 4,7 ). We noted that more VVI was released than was ATV, IVI, or TLV in the $A \beta_{41}$ product line. Following the stepwise-processing model, the relatively high level of VVI released would be explained by a contribution from an additional 

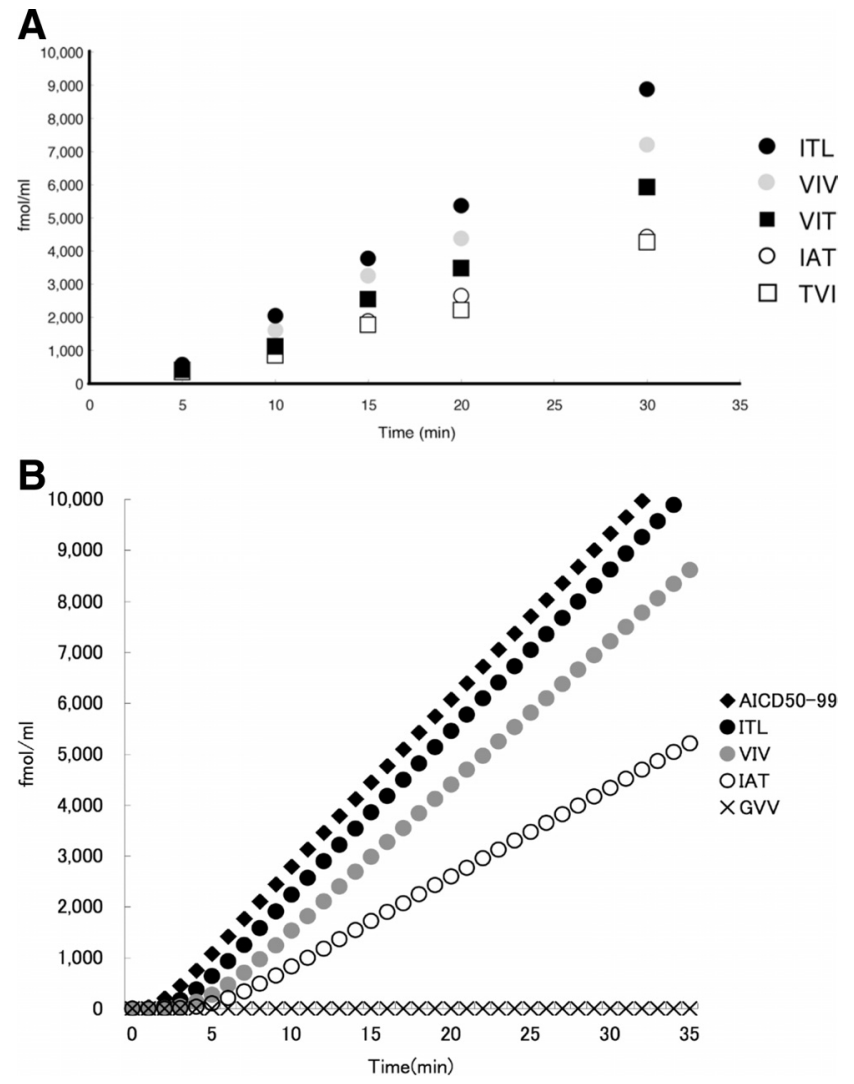

Figure 8. $\quad \boldsymbol{A}$, The very early phase of the tripeptide-release reaction. The reaction mixture was sampled at 5-30 min and subjected to LC-MS/MS analysis for tripeptide quantification. Approximately $245 \mu \mathrm{l}$ of $0.25 \%$ CHAPSO buffer containing $0.1 \%$ phosphatidylcholine was preincubated at $37^{\circ} \mathrm{C}$. The CHAPSO buffer that was kept at $37^{\circ} \mathrm{C}$ was added to the $\gamma$-secretasebound protein A-Sepharose $(\sim 20 \mu l)$ in an Eppendorf tube that had been kept on ice, and the mixture was incubated at $37^{\circ} \mathrm{C}$ for $5 \mathrm{~min}$. The reaction was started by addition of the substrate, (99-FLAG $(\sim 5 \mu l)$ that had been kept on ice, and the reaction mixture continued with agitation in a chamber kept at $37^{\circ} \mathrm{C}$. Extrapolation of each time line for each tripeptide appears to intercept the same point on the $x$-axis, indicating that the reactions start after a 3-4 min induction period. The corrections for the peptides were the same as above (see the legends to Figs. 3,4). Note that the relationships ITL $>$ VIV $>$ IAT and VIT $>$ TVI were maintained up to 10 min. Black circles, ITL; gray circles, VIV; white circles, IAT; black squares, VIT; white squares, TVI. $B$, Tripeptide-release kinetics simulated based on the stepwise-processing model. A set of parameters were optimized using genetic algorithm to fit the experimental data in Figure $8 \mathrm{~A}$ (supplemental Table $\$ 4$, available at www.jneurosci.org as supplemental material). The simulation shows that there are induction periods for the generation of AICD50-99 as well as for that of tripeptides. After induction periods, 3-4 min for tripeptides and $\sim 2$ min for AICD49-99, the reactions proceeded in a linear manner up to $6 \mathrm{~h}$ (data not shown).

product line(s). For example, the release of VIT in the $\mathrm{A} \beta_{42}$ product line, followed by the release of tetrapeptide ATVI, would lead to $A \beta_{41}$. In any case, the production of $A \beta_{38}$ would be contributed not only from $\mathrm{A} \beta_{42}$ but also from $\mathrm{A} \beta_{41}$ by releasing VVI (see below). Thus, quantification of VVIA alone may underestimate the level of $\mathrm{A} \beta_{38}$. Such unusual cleavage that does not follow the tripeptide (or sometimes tetrapeptide) rule may tend to occur in the CHAPSO-reconstituted system that allows free collision between the enzyme and substrate. Conversely, membrane integrity would enhance these minor processes and production of smaller $\mathrm{A} \beta \mathrm{s}$ that are barely detectable in the reconstituted system. In relation to this unusual cleavage, we note that intracellular generation of $\mathrm{A} \beta_{49}$ or $\mathrm{A} \beta_{48}$ induced by transfection leads to production of small but significant amounts of $\mathrm{A} \beta_{40}$ or $\mathrm{A} \beta_{42}$, respectively. This suggests that the two product lines are not completely independent and may have some junctions. It should be also noted that the present theoretical model does not take into account minor and unusual cleavage(s) or interdependence between the product lines.

The stepwise-processing model has the inherent characteristic that the difference in the levels of tripeptide released is equal to the amount of $\mathrm{A} \beta$ produced (Fig. 5). Only the levels of the final products, $\mathrm{A} \beta_{40}$ and $\mathrm{A} \beta_{38}$, are equal to the released amounts of tripeptides and tetrapeptides, IAT and VVIA (but see above), respectively. Because the levels of other $\mathrm{A} \beta$ s represent the difference of tripeptide (and tetrapeptide) released, it is possible that, even if the trimming activity of $\gamma$-secretase increases markedly, the level of $\mathrm{A} \beta$ of interest is low. Conversely, the level of $\mathrm{A} \beta$ of interest may increase despite suppressed activity of $\gamma$-secretase. Accordingly, the levels of $A \beta$ s do not reflect the cleavage activity at each step. One noteworthy example is the effect of sulindac sulfide on the $\gamma$-secretase assay system (Fig. 7), i.e., the cleaving activities of $\gamma$-secretase do not significantly alter. Nonetheless, the levels of produced $\mathrm{A} \beta_{43}$ and $\mathrm{A} \beta_{42}$ decreased, whereas the tetrapeptide VVIA tended to increase. Because the levels of $A \beta_{40}$ stayed constant, only the level of $\mathrm{A} \beta_{42}$ appeared to decrease, leading to the prevailing view that sulindac sulfide is selective for the $\mathrm{A} \beta_{42}$ product line. However, our results (Fig. 7) suggest that this may not be correct. It is possible that sulindac sulfide affects the final processes of $\mathrm{A} \beta$ generation, by enhancing of cleaving of IAT and VVIA, which decreased the levels of $\mathrm{A} \beta_{43}$ and $\mathrm{A} \beta_{42}$.

This model explains why it was difficult for us to find possible intermediates within the lysates because these intermediates are characterized by their very low levels in the cell. If an intermediate is a good substrate for $\gamma$-secretase, it would be cleaved promptly, generating another intermediate and leaving nothing behind. Thus, only intermediates relatively resistant to $\gamma$-secretase would be easy to detect in the cell. Why we often detect $A \beta_{46}$ in the cell would be most likely attributable to its relative resistance to VIVcleaving activity of $\gamma$-secretase within the membrane. In contrast, in the CHAPSO-reconstituted system, $\mathrm{A} \beta_{46}$ was very susceptible to this cleaving activity, and only a very low level of $A \beta_{46}$ was produced, which was undetectable by Western blotting.

We do not know why the CHAPSO-reconstituted system processes $\beta$ CTF up to $\mathrm{A} \beta_{40 / 38}$ but no further to smaller species, neither do we know how the substrate is translocated on the catalytic site of the $\gamma$-secretase complex during the successive cleavage process. Apparently, this step is energy independent, because the cleavage does not require ATP or GTP. Interestingly, an exogenously added $\mathrm{A} \beta_{46}, \mathrm{~A} \beta_{48}$, or $\mathrm{A} \beta_{49}$ is never processed to smaller species by the CHAPSO-reconstituted $\gamma$-secretase (data not shown). Presumably, a distinct cleavage-sensitive conformation would be required for long $A \beta$ s to bind to the $\gamma$-secretase complex. This may relate to the previous observation that longer $\mathrm{A} \beta$ species generated by transfection are easily processed, and smaller $\mathrm{A} \beta$ species are more difficult to process (Funamoto et al., 2004).

We do not have a proper explanation about why $\gamma$-secretase cleaves out VVIA to produce $\mathrm{A} \beta_{38}$ but never VIA to produce $\mathrm{A} \beta_{39}$ in this reconstituted system. Other cleavage sites definitely follow the tripeptide rule and fit well within the $\alpha$-helical model proposed originally by Lichtenthaler et al. (2002). When one looks at the sequence of TMD of $\beta \mathrm{CTF}$, bulky hydrophobic residues except Ala42 are filled between the $\varepsilon$ - and $\gamma$-cleavage sites up to the GG portion in which the smallest residue appears serially. The $\alpha$-helical surface of the TMD may be shallow grooved in this portion, which may provide a stronger signal for binding to the catalytic sites of $\gamma$-secretase. In other words, the $\gamma$-secretase may prefer to cleave near the less hindered $\mathrm{G}-\mathrm{V}$ amide bond rather than the more hindered $\mathrm{V}-\mathrm{V}$ amide bond. 
Although the present CHAPSO-reconstituted system demonstrates the framework of $\gamma$-secretase cleavage, stepwise processing, it lacks some features observed in cell-free system or cultured cells. First, remarkable accumulation of $\mathrm{A} \beta_{43 / 46}$ in cultured cells treated by DAPT cannot be seen in the CHAPSO-reconstituted system. Presumably, differential sensitivity of $\gamma$-secretase/A $\beta$ complex to effective concentrations of DAPT in the cell could explain the phenomenon. The $\gamma$-secretase $/ \mathrm{A} \beta_{43}$ complex is more sensitive to DAPT than is the $\gamma$-seretase $/ \mathrm{A} \beta_{46}$ complex. Alternatively, the catalytic sites in the membrane of $\gamma$-seretase/A $\beta_{43}$ would be located in the outer portion of the membrane and would thus be more sensitive to DAPT, whereas the $\gamma$-secretase/ $\mathrm{A} \beta_{46}$ complex would be located in the inner portion of the membrane and be less sensitive to DAPT. The CHAPSO-reconstituted system has no membrane barrier, resulting in loss of differential sensitivities. Second, more $\mathrm{A} \beta_{40}$ than $\mathrm{A} \beta_{42}$ is usually produced in the cells or cell-free system (Fig. 2). In contrast, the CHAPSOreconstituted system generates similar levels of $A \beta_{40}, A \beta_{42}$, and $\mathrm{A} \beta_{43}$ (Kakuda et al., 2006). The two product lines are predominant and substantially independent; this comes from abundance of $\mathrm{A} \beta_{48}$ relative to $\mathrm{A} \beta_{49}$ in $\varepsilon$-cleaved products in the CHAPSOreconstituted system. This suggests that the cleavage at the carboxyl side of Thr48 would be enhanced in the CHAPSOreconstituted system compared with the cell-free system. It is possible that membrane integrity or some regulatory protein factors suppress the generation of $\mathrm{A} \beta_{48}$ and thus the generation of $\mathrm{A} \beta_{42}$. LC-MS/MS analysis of the raft system (Yagishita et al., 2006) may provide insight into the characteristics of $\gamma$-secretase embedded in the membrane.

Although the CHAPSO-reconstituted system has some inherent problems, we believe that this stepwise-processing model provides the framework for investigating the cleavage by $\gamma$-secretase. The characteristics revealed here should be considered when studying the structure-function relationship of $\gamma$-secretase complex (Lazarov et al., 2006; Osenkowski et al., 2009).

\section{References}

Beher D, Wrigley JD, Owens AP, Shearman MS (2002) Generation of C-terminally truncated amyloid- $\beta$ peptides is dependent on $\gamma$-secretase activity. J Neurochem 82:563-575.

Eriksen JL, Sagi SA, Smith TE, Weggen S, Das P, McLendon DC, Ozols VV, Jessing KW, Zavitz KH, Koo EH, Golde TE (2003) NSAIDs and enantiomers of flurbiprofen target $\gamma$-secretase and lower $\mathrm{A} \beta 42$ in vivo. J Clin Invest 112:440-449.

Funamoto S, Morishima-Kawashima M, Tanimura Y, Hirotani N, Saido TC,
Ihara Y (2004) Truncated carboxyl-terminal fragments of $\beta$-amyloid precursor protein are processed to amyloid $\beta$-proteins 40 and 42 . Biochemistry 43:13532-13540.

Iwatsubo T, Odaka A, Suzuki N, Mizusawa H, Nukina N, Ihara Y (1994) Visualization of $A \beta 42(43)$ and $A \beta 40$ in senile plaques with end-specific $\mathrm{A} \beta$ monoclonals: evidence that an initially deposited species is $\mathrm{A} \beta$ 42(43). Neuron 13:45-53.

Kakuda N, Funamoto S, Yagishita S, Takami M, Osawa S, Dohmae N, Ihara Y (2006) Equimolar production of amyloid $\beta$-protein and amyloid precursor protein intracellular domain from $\beta$-carboxyl-terminal fragment by $\gamma$-secretase. J Biol Chem 281:14776-14786.

Kukar T, Murphy MP, Eriksen JL, Sagi SA, Weggen S, Smith TE, Ladd T, Khan MA, Kache R, Beard J, Dodson M, Merit S, Ozols VV, Anastasiadis PZ, Das P, Fauq A, Koo EH, Golde TE (2005) Diverse compounds mimic Alzheimer disease-causing mutations by augmenting A $\beta 42$ production. Nat Med 11:545-550.

Lazarov VK, Fraering PC, Ye W, Wolfe MS, Selkoe DJ, Li H (2006) Electron microscopic structure of purified, active $\gamma$-secretase reveals an aqueous intramembrane chamber and two pores. Proc Natl Acad Sci U S A 103: $6889-6894$.

Lichtenthaler SF, Beher D, Grimm HS, Wang R, Shearman MS, Masters CL, Beyreuther K (2002) The intramembrane cleavage site of the amyloid precursor protein depends on the length of its transmembrane domain. Proc Natl Acad Sci U S A 99:1365-1370.

Osawa S, Funamoto S, Nobuhara M, Wada-Kakuda S, Shimojo M, Yagishita S, Ihara Y (2008) Phosphoinositides suppress $\gamma$-secretase in both the detergent-soluble and -insoluble states. J Biol Chem 283:19283-19292.

Osenkowski P, Li H, Ye W, Li D, Aeschbach L, Fraering PC, Wolfe MS, Selkoe DJ, $\mathrm{LiH}$ (2009) Cryoelectron microscopy structure of purified $\gamma$-secretase at 12 A resolution. J Mol Biol 385:642-652.

Qi-Takahara Y, Morishima-Kawashima M, Tanimura Y, Dolios G, Hirotani N, Horikoshi Y, Kametani F, Maeda M, Saido TC, Wang R, Ihara Y (2005) Longer forms of amyloid $\beta$-protein: implications for the mechanism of intramembrane cleavage by $\gamma$-secretase. J Neurosci 25:436-445.

Sato T, Dohmae N, Qi Y, Kakuda N, Misonou H, Mitsumori R, Maruyama H, Koo EH, Haass C, Takio K, Morishima-Kawashima M, Ishiura S, Ihara Y (2003) Potential link between amyloid $\beta$-protein 42 and C-terminal fragment $\gamma$ 49-99 of $\beta$-amyloid precursor protein. J Biol Chem 278: 24294-24301.

Weggen S, Eriksen JL, Das P, Sagi SA, Wang R, Pietrzik CU, Findlay KA, Smith TE, Murphy MP, Bulter T, Kang DE, Marquez-Sterling N, Golde TE, Koo EH (2001) A subset of NSAIDs lower amyloidogenic A $\beta 42$ independently of cyclooxygenase activity. Nature 414:212-216.

Yagishita S, Morishima-Kawashima M, Tanimura Y, Ishiura S, Ihara Y (2006) DAPT-induced intracellular accumulations of longer amyloid $\beta$-proteins: further implications for the mechanism of intramembrane cleavage by $\gamma$-secretase. Biochemistry 45:3952-3960.

Yagishita S, Morishima-Kawashima M, Ishiura S, Ihara Y (2008) A $\beta 46$ is processed to $\mathrm{A} \beta 40$ and $\mathrm{A} \beta 43$, but not to $\mathrm{A} \beta 42$, in the low density membrane domains. J Biol Chem 283:733-738. 